\title{
Shortened TDP43 isoforms upregulated by neuronal hyperactivity drive TDP43 pathology in ALS
}

\author{
Kaitlin Weskamp, ${ }^{1,2}$ Elizabeth M. Tank, ${ }^{1}$ Roberto Miguez, ${ }^{1}$ Jonathon P. McBride, ${ }^{1,3}$ Nicolás B. Gómez, ${ }^{1,3}$ Matthew White, ${ }^{4}$ \\ Ziqiang Lin, ${ }^{4}$ Carmen Moreno Gonzalez, ${ }^{5}$ Andrea Serio, ${ }^{5}$ Jemeen Sreedharan, ${ }^{4}$ and Sami J. Barmada ${ }^{1,2,3}$
}

'Department of Neurology, ${ }^{2}$ Neuroscience Graduate Program, and ${ }^{3}$ Cellular and Molecular Biology Program, Michigan Medicine, University of Michigan, Ann Arbor, Michigan, USA. ${ }^{4}$ Department of Basic and Clinical Neuroscience and ${ }^{5}$ Centre for Craniofacial and Regenerative Biology, King's College London, London, United Kingdom.

\begin{abstract}
Cortical hyperexcitability and mislocalization of the RNA-binding protein TDP43 are highly conserved features in amyotrophic lateral sclerosis (ALS). Nevertheless, the relationship between these phenomena remains poorly defined. Here, we showed that hyperexcitability recapitulates TDP43 pathology by upregulating shortened TDP43 (sTDP43) splice isoforms. These truncated isoforms accumulated in the cytoplasm and formed insoluble inclusions that sequestered full-length TDP43 via preserved $\mathrm{N}$-terminal interactions. Consistent with these findings, sTDP43 overexpression was toxic to mammalian neurons, suggesting neurodegeneration arising from complementary gain- and loss-of-function mechanisms. In humans and mice, sTDP43 transcripts were enriched in vulnerable motor neurons, and we observed a striking accumulation of sTDP43 within neurons and glia of ALS patients. Collectively, these studies uncover a pathogenic role for alternative TDP43 isoforms in ALS, and implicate sTDP43 as a key contributor to the susceptibility of motor neurons in this disorder.
\end{abstract}

\section{Introduction}

Amyotrophic lateral sclerosis (ALS) is a neurodegenerative disorder in which the progressive loss of motor neurons results in paralysis and respiratory failure (1). There is no disease-modifying therapy for ALS, and its heterogeneous biochemical, genetic, and clinical features complicate the identification of therapeutic targets. However, the cytoplasmic mislocalization and accumulation of TDP43 (TAR DNA-binding protein of $43 \mathrm{kDa}$ ), a nuclear RNA-binding protein integrally involved in RNA metabolism, is observed in more than $90 \%$ of individuals with ALS (2). Moreover, while mutations in the gene encoding TDP43 (TARDBP) only account for $2 \%-5 \%$ of ALS cases, mutations in several other ALS-associated genes including C9ORF72 (3), ANG (4), TBK1 (5), PFN1 (6), UBQLN2 (7), VCP (8), and HNRNPA2B1 (9) result in TDP43 pathology.

TDP43 is an essential protein involved in several RNA processing events, including splicing, translation, and degradation. In keeping with these fundamental functions, TDP43 levels and localization are tightly regulated and critical for cell health. TDP43-knockout animals exhibit neurodegeneration and behavioral deficits (10-13), while TDP43 overexpression results in neurodegeneration in primary neuron $(14,15)$, mouse $(16,17)$, rat $(18$, 19), Drosophila $(20,21)$, zebrafish $(22,23)$, and primate models $(24,25)$. Furthermore, mislocalization of TDP 43 to the cytoplasm is sufficient to drive cell death (14). Taken together, these observations suggest that even small changes in TDP43 levels and localization are highly predictive of neurodegeneration.

Conflict of interest: The authors have declared that no conflict of interest exists. Copyright: (c) 2020, American Society for Clinical Investigation.

Submitted: June 10, 2019; Accepted: November 6, 2019; Published: January 27, 2020.

Reference information: / Clin Invest. 2020;130(3):1139-1155.

https://doi.org/10.1172/JCl130988
Hyperexcitability, or an increase in neuronal activity, is also a conserved feature in both familial and sporadic ALS (sALS) (26). Cortical hyperexcitability precedes symptom onset in some cases (26), and the degree of motor neuron excitability is a strong predictor of disease progression $(27,28)$. Such hyperexcitability arises from a loss of cortical inhibition $(26,29-33)$ in combination with intrinsic differences in channel expression, content, and activity within motor neurons themselves $(26,28,34,35)$. Emphasizing the contribution of hyperexcitability to disease, riluzole -1 of 2 available therapies for ALS - is a sodium channel antagonist that partially rescues hyperexcitability (36). Animal models of ALS recapitulate key features of hyperexcitability (37-39), including an increase in motor neuron activity that precedes the onset of motor deficits $(37,39,40)$ and reduced activity following treatment with riluzole (41). Hyperexcitability is also observed in induced pluripotent stem cell-based (iPSC-based) ALS models $(42,43)$, though other reports suggest that it may be a transient or developmental phenomenon $(43,44)$.

Despite the prevalence of both TDP43 pathology and hyperexcitability in ALS, the relationship between these phenomena remains poorly defined. Here, we utilize an iPSC-derived neuron (iNeuron) model system to demonstrate that hyperexcitability drives TDP43 pathology characteristic of ALS via the upregulation of atypical, shortened TDP43 isoforms. Using multiple model systems and human postmortem material, we show that these unusual isoforms are exported from the nucleus, form insoluble cytoplasmic inclusions, are neurotoxic, and are enriched in ALS patient tissue, thereby directly implicating alternative TDP43 isoforms in ALS pathogenesis.

\section{Results}

TDP43 is regulated by neuronal activity. To investigate disease mechanisms related to hyperexcitability in human neurons, we 
A

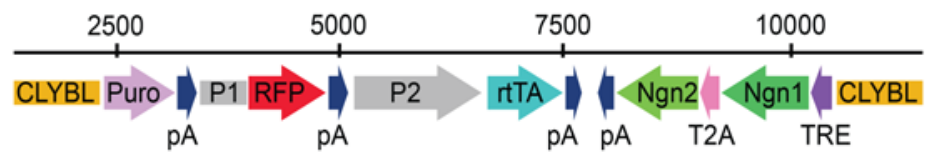

C
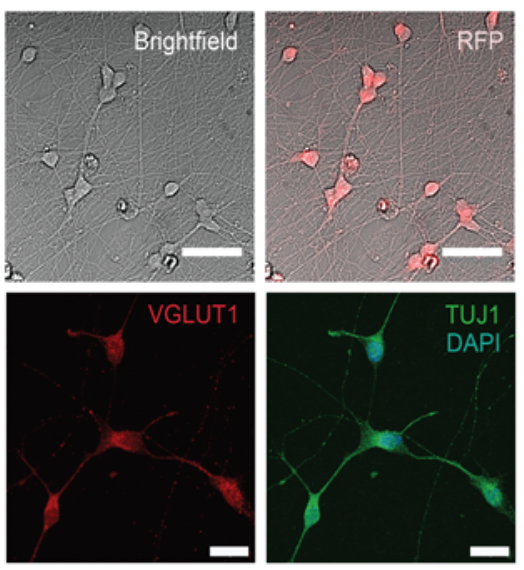

D

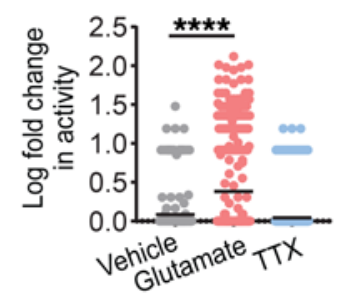

E

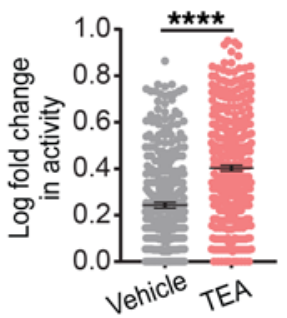

B

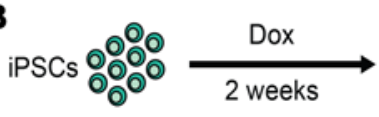

$\mathbf{F}$
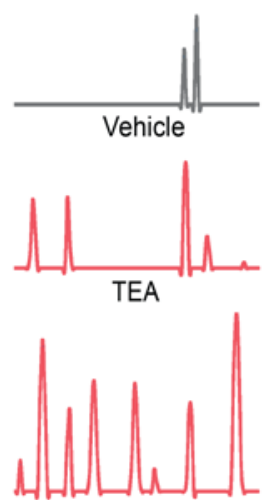

Glutamate

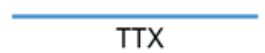

H
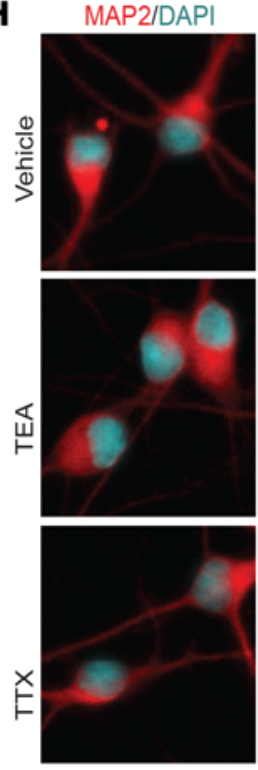

J
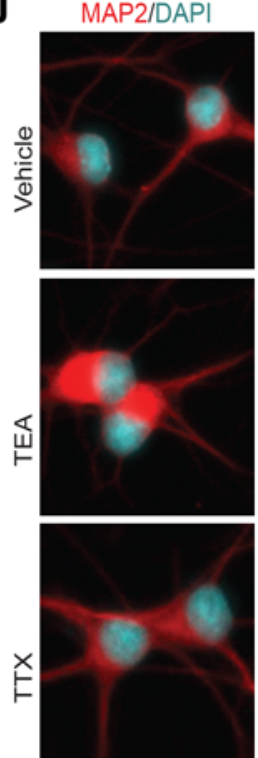

$\mathrm{N}$-Term TDP43
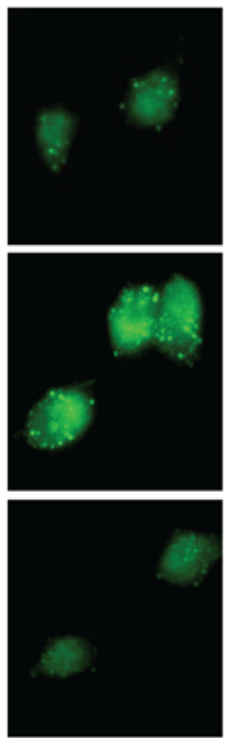

C-Term TDP43
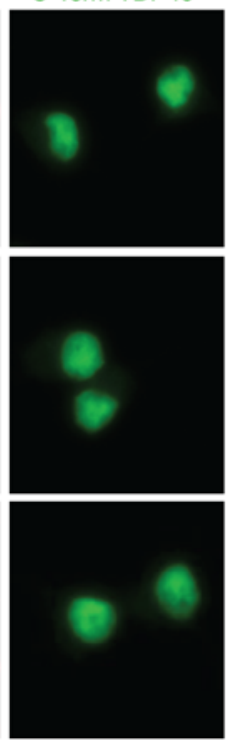

Merge
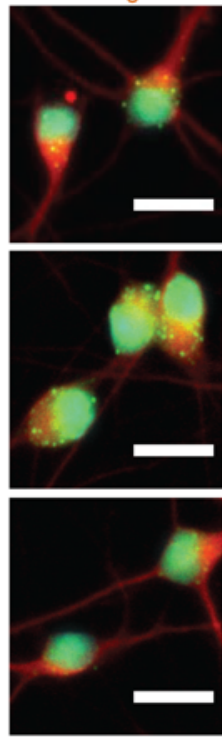

Merge
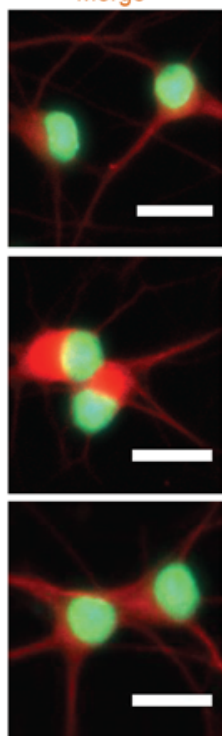

I $\quad$ Vehicle $(n=110)$

- $\operatorname{TEA}(4 \mathrm{mM})(n=113)$

चTX $(2 \mu \mathrm{M})(n=96)$

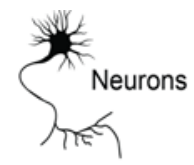

$\mathbf{G}$

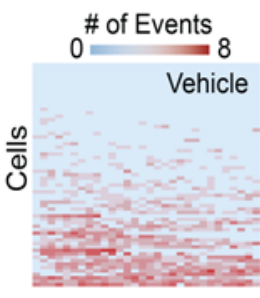

TEA

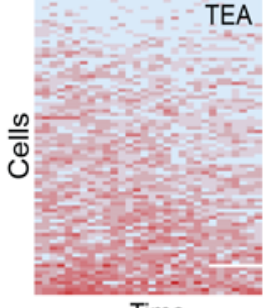

Time
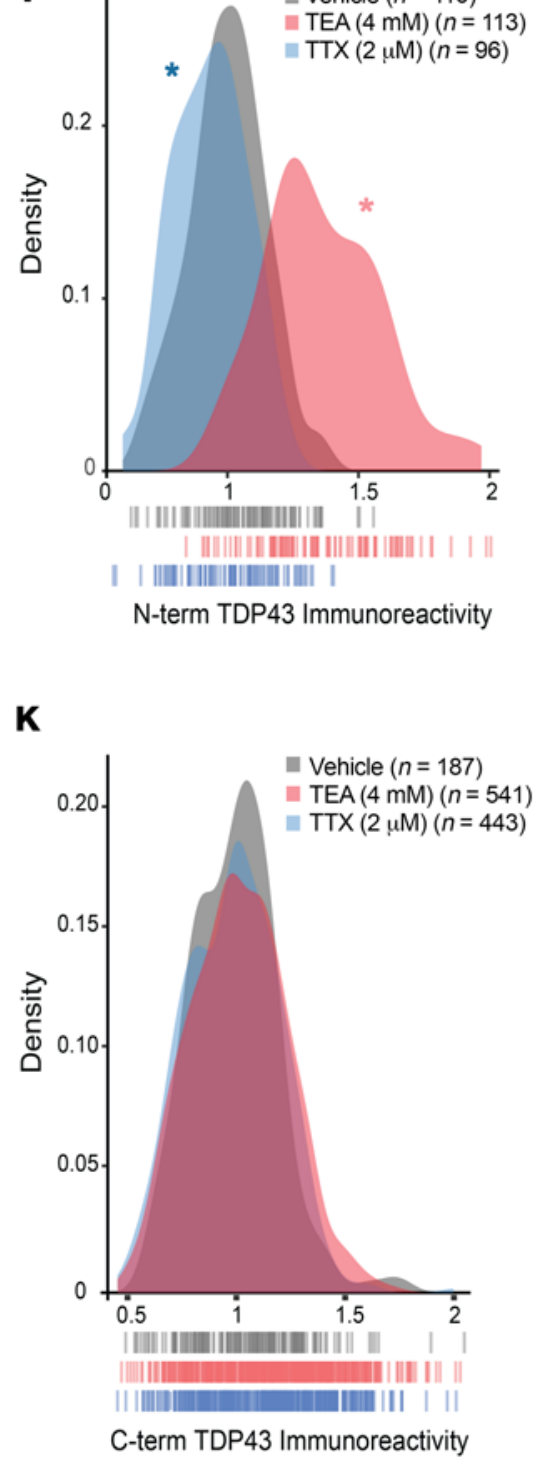

$\mathrm{N}$-term TDP43 Immunoreactivity

$\mathbf{K}$ 
Figure 1. Hyperexcitability drives TDP43 accumulation in human iNeurons. (A) Schematic of the cassette used to integrate Ngn1 and Ngn2 into the CLYBL safe harbor locus under a doxycycline-inducible (Tet-on) promoter. CLYBL, targeting sequence; Puro, puromycin resistance gene; $\mathrm{PA}$, poly-A tail; P1, P2, promoters; RFP, mCherry; rtTA, reverse tetracycline-controlled transactivator; $\mathrm{Ngn} 1$ and -2 , neurogenin 1 and 2; T2A, self-cleaving peptide; TRE, tetracycline response element. (B) Timeline depicting the differentiation of iPSCs into forebrain-like neurons within 2 weeks of doxycycline addition. (C) The resultant neurons are RFP positive and express the neuronal markers VGLUT1 and TUJ1. (D) Spontaneous neuronal activity visualized by the $\mathrm{Ca}^{2+}$ reporter gCaMP6f at 2 weeks. Activity was pharmacologically modulated with bath application of glutamate or TTX. Vehicle $n$ $=257$, glutamate $n=327, \operatorname{TTX} n=403$, stratified among 3 replicates. ${ }^{* * *} P$ $<0.0001$ by 1-way ANOVA with Dunnett's post hoc test. (E) Treatment with TEA significantly increased neuronal activity. Vehicle $n=312$, TEA $n=369$, stratified among 3 replicates. ${ }^{* * *} P<0.0001$ by 2 -tailed $t$ test. (F) Example traces depicting changes in gCaMP6f fluorescence for each condition. (C) Heatmaps depicting global changes in activity. Each row represents 1 neuron, and each column represents a 20 -second observation window. Thirty intervals were collected over a 12-hour period. Box color indicates the relative firing rate of each cell at each time point, ranging from low (blue) to high (red). (H) N-terminal TDP43 immunoreactivity was increased in TEA-treated iNeurons and decreased in TTX-treated iNeurons. (I) Density plot depicting the change in TDP43 immunoreactivity between conditions. Vehicle $n=110$, TEA $n=113$, TTX $n=96,2$ replicates; vertical lines indicate single neurons. ${ }^{*} P<0.05$ by Kolmogorov-Smirnov test. (J) No change in TDP43 abundance was detected using an antibody directed against the C-terminus. (K) Density plot depicting the change in C-terminal TDP43 immunoreactivity between conditions. Vehicle $n=187$, TEA $n=541$, TTX $n=443$, 2 replicates; vertical lines indicate single neurons. No significant differences as determined by the Kolmogorov-Smirnov test. Scale bars: 50 $\mu \mathrm{m}$ (top), $20 \mu \mathrm{m}$ (bottom) (C); $20 \mu \mathrm{m}$ (H and J).

established an iNeuron model. Transcription activator-like endonucleases (TALENs) specific for the CLYBL safe harbor locus were used to introduce the transcription factors neurogenin 1 and 2 (Ngn1 and -2) under a doxycycline-inducible (dox-inducible) promoter (Figure 1A). Expression of Ngn1 and -2 is sufficient to drive the rapid differentiation of iPSCs into iNeurons that display immunocytochemical and electrophysiological properties of glutamatergic, excitatory forebrain-like neurons (45-47) (Figure 1B). Consistent with this, within 2 weeks of dox addition iNeurons adopt a neuronal morphology and stain positive for the neuronal markers VGLUT1 and TUJ1 (Figure 1C). We further validated the maturity of neurons differentiated in this manner using an iPSC line that stably expresses the fluorescent calcium indicator gCaMP6f in addition to dox-inducible Ngn1 and -2 (48). Because time-dependent changes in gCaMP6f fluorescence correlate with action potentials, we monitored neuronal activity indirectly and noninvasively in iNeurons by fluorescence microscopy. Two to 3 weeks following dox addition, iNeurons displayed a low level of spontaneous activity that was significantly increased with bath application of the neurotransmitter glutamate or the potassium channel blocker tetraethylammonium (TEA) (Figure 1, D-F). Conversely, activity was inhibited by application of the sodium channel blocker tetrodotoxin (TTX). Although glutamate dramatically increased neuronal activity, it proved to be toxic even at low doses (data not shown). In comparison, iNeurons treated with TEA showed a smaller, sustained increase in activity without significant cell death (Figure 1G). Thus, TEA and TTX were utilized in subsequent studies of activity-dependent TDP43 regulation.
To explore a potential connection between hyperexcitability and TDP43 pathology, we pharmacologically stimulated or blocked activity in iNeuron cultures and then examined changes in TDP43 levels via immunocytochemistry (ICC) using an antibody directed against the TDP43 N-terminus. To quantitatively gauge differences in neuronal TDP43, we utilized MAP2 staining to generate cellular regions of interest (ROIs), and measured TDP43 immunoreactivity within individual neurons. TEA-treated iNeurons showed a significant increase in TDP43 immunoreactivity while TTX-treated iNeurons exhibited a reduction, suggesting a bidirectional relationship between TDP43 abundance and neuronal activity (Figure 1, H and I). An analogous relationship was observed in rodent primary mixed cortical neurons treated with glutamate or the GABA receptor antagonist bicuculline (Supplemental Figure 1A; supplemental material available online with this article; https://doi.org/10.1172/JCI130988DS1).

Unexpectedly, when we repeated these studies using an antibody directed against the TDP43 C-terminus, we failed to identify significant activity-dependent changes in protein abundance (Figure 1, J and $\mathrm{K}$ ), and also noted prominent differences in subcellular TDP43 distribution identified by each antibody (Figure $1, \mathrm{H}$ and $\mathrm{J}$ ). Immunostaining with $\mathrm{N}$-terminal antibodies revealed punctate, cytoplasmic TDP43 superimposed on nuclear TDP43 in both iNeurons (Figure 1H) and rodent primary mixed cortical neurons treated with bicuculline (Supplemental Figure 1B). However, only nuclear TDP43 was detectable using C-terminal TDP43 antibodies (Figure 1J). A survey of commercially available antibodies with known epitopes revealed similar trends in localization; antibodies that recognize the TDP $43 \mathrm{~N}$-terminus are more likely to display nuclear and cytoplasmic staining patterns (49-51), while antibodies specific for the C-terminus primarily show nuclear TDP43 $(52,53)$.

Given the variability in antibody specificity and potential difficulties in reproducing results using different antibodies (54, 55 ), we validated our findings by fluorescently labeling native TDP43 in iPSCs using CRISPR/Cas9 genome engineering. To minimize off-target effects, we used a dual-nickase approach (56) to fuse the green fluorescent protein Dendra2 to either the N-terminus (D2-TDP43) or the C-terminus (TDP43-D2) of endogenous TDP43 in human iPSCs (Figure 2A and Supplemental Figure 2). D2-TDP43 and TDP43-D2 iPSCs were differentiated into iNeurons as described above (Figure 2, B and C), and neuronal activity was pharmacologically stimulated or blocked using TEA or TTX, respectively. After 48 hours, we visualized native Dendra2-labeled TDP43 by fluorescence microscopy, noting a bidirectional relationship between D2-TDP43 abundance and neuronal activity (Figure 2D) that was nearly identical to what we observed using antibodies that recognize the TDP43 N-terminus (Figure 1, H and I). In comparison, there were no significant activity-dependent changes in TDP43-D2 (Figure 2E), consistent with our inability to detect changes upon staining with antibodies raised against the TDP43 C-terminus (Figure 1, J and $\mathrm{K}$ ). These data provide convincing evidence for TDP43 species harboring the $\mathrm{N}$ - but not the $\mathrm{C}$-terminus that are regulated by neuronal activity. Additionally, the distinctive TDP43 distribution patterns revealed by N- and C-terminusreactive antibodies were reflected by the localization of Den- 
A
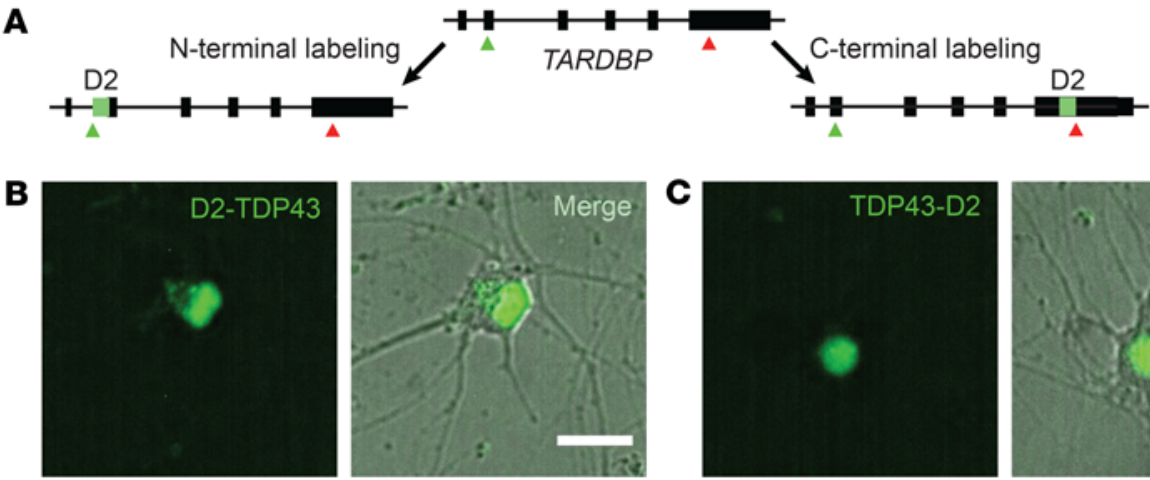

D

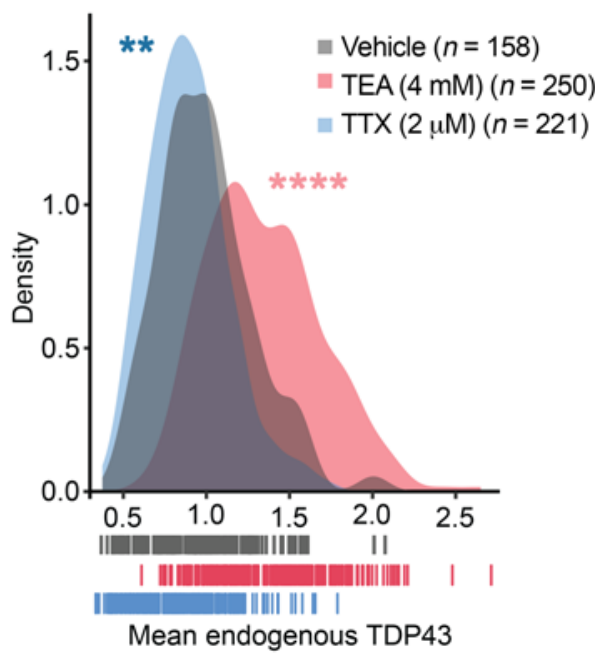

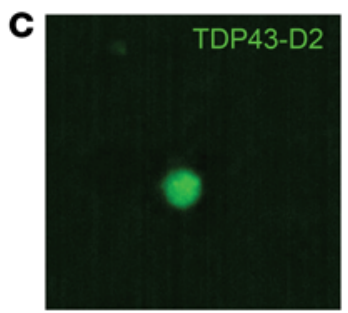

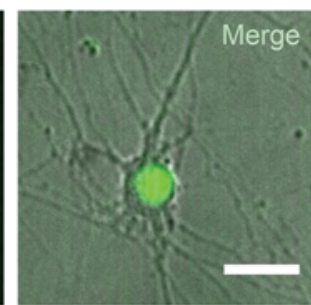

E

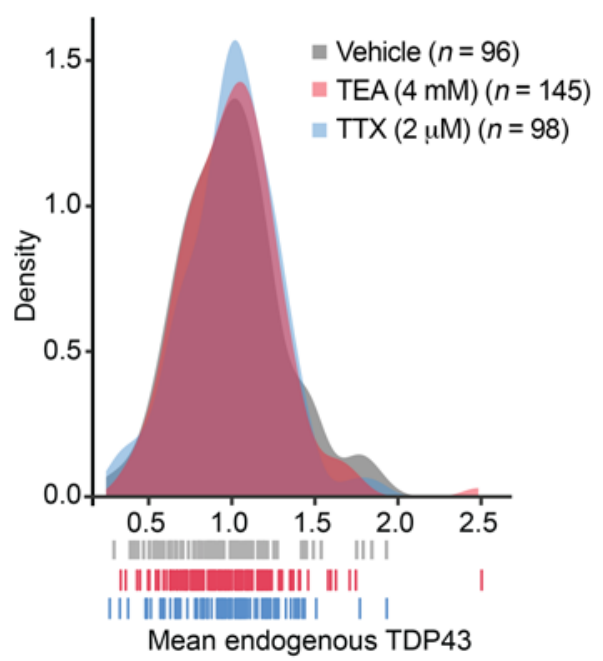

Figure 2. TDP43 species harboring the $\mathrm{N}$ - but not the $\mathrm{C}$-terminus are regulated by neuronal activity. (A) Strategy for labeling native TDP43 in human iPSC-derived neurons using CRISPR/Cas9. Dendra2 (D2, green) was inserted $3^{\prime}$ relative to the TARDBP start codon (green arrow) or $5^{\prime}$ relative to the conventional stop codon (red arrow), enabling fluorescent labeling of the TDP43 N- or C-terminus, respectively. In iNeurons, N-terminally tagged TDP43 (B, D2-TDP43) appeared both nuclear and cytoplasmic in distribution, while C-terminally tagged TDP43 (C, TDP43-D2) was primarily nuclear. (D) Density plot depicting the fluorescence intensity of D2-TDP43 upon application of vehicle $(n=158)$, TEA $(n=250)$, or TTX $(n=221)$. (E) Density plot depicting the fluorescence intensity of TDP43-D2 with addition of vehicle $(n=96)$, TEA $(n=145)$, or $\operatorname{TTX}(n=98)$. In $\mathbf{D}$ and $\mathbf{E}$, vertical lines indicate individual neurons from 2 replicates. ${ }^{* *} P<0.01,{ }^{* * * *} P$ $<0.0001$ by Kolmogorov-Smirnov test. Scale bars: $20 \mu \mathrm{m}$ (B and C). dra2-tagged native TDP43; D2-TDP43 appeared both cytoplasmic and nuclear (Figure 2B), while the distribution of TDP43-D2 was limited to the nucleus (Figure 2C).

Collectively, these results suggest that neuronal activity elicits an increase in cytoplasmic TDP43 that lacks a C-terminus. In contrast with what we observed with N-terminal TDP43, there was no reciprocal activity-dependent change in C-terminal TDP43 abundance or localization by ICC, and we failed to observe any differences in C-terminally labeled TDP43-D2 upon addition of TEA or TTX, arguing against a cleavage event. However, previous studies demonstrated that neuronal activity regulates the abundance of similar RNA-binding proteins through alternative splicing $(57,58)$. We therefore considered the possibility that activity gives rise to distinct TDP43 isoforms through alternative splicing.

Hyperexcitability drives TARDBP alternative splicing. Using available RNA sequencing (RNA-seq) data obtained from human cell lines (59), we identified 2 alternatively spliced $T A R D B P$ isoforms predicted to encode C-terminally truncated or shortened TDP43 (sTDP43) isoforms (Figure 3A). Identical sTDP43 splice isoforms (TDP-S6 and TDP-S7) were detected in previous studies of TARDBP splicing $(60,61)$. Both STDP43-specific splice donors are located within TARDBP exon 6 and differ by only $9 \mathrm{bp}$; each utilizes an identical splice acceptor within the TARDBP $3^{\prime}$ untranslated region (UTR), thereby eliminating the majority of exon 6 (Figure 3B). We designed primers specific for both sTDP43 splice junctions as well as fulllength TDP43 (flTDP43) utilizing the canonical termination codon within exon 6, and performed quantitative reverse transcription PCR (qRT-PCR) to examine changes in splice isoform abundance in vehicle-, TEA-, or TTX-treated human iNeurons. sTDP43 isoforms were not only detectable in iNeurons, but also significantly upregulated by treatment with TEA (Figure 3C); while there was a trend toward downregulation with TTX, this did not reach statistical significance. These results suggest that the bidirectional change in N-terminal TDP43 observed in TEA- or TTX-treated iNeurons may be due to altered expression of sTDP43 transcript isoforms. Transcripts encoding flTDP43 were significantly more abundant than the sTDP43 isoforms, and demonstrated a similar upregulation with TEA and downward trend with TTX (Figure 3, C and D). Thus, although all TARDBP transcript variants increase with neuronal activity, only sTDP43 isoforms demonstrate corresponding changes at the protein level, perhaps due to selective autoregulation or nuclear retention of spliced full-length transcripts (61-63).

The 2 sTDP 43 transcripts (sTDP43-1 and -2) encode proteins that differ by only 3 amino acids, and both lack residues that correspond to the entirety of the glycine-rich domain (residues 281-414 of flTDP43) (64). Usage of the common splice acceptor for sTDP43-1 and -2 located within the TARDBP 3'UTR results in the inclusion of a new exon encoding a unique 18-amino acid C-terminus not found in flTDP43 (Figure 3E). These splicing events and the distinct sTDP43 C-terminus are highly conserved at both the transcript (Supplemental Table 1) and protein (Supplemental Table 2) levels in humans, nonhuman primates, and 
A

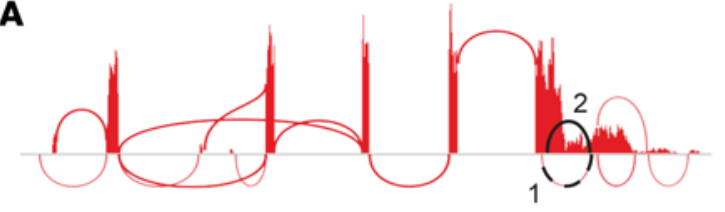

B
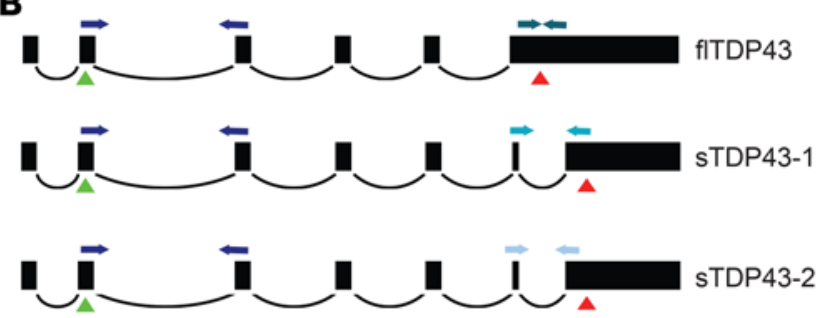

D

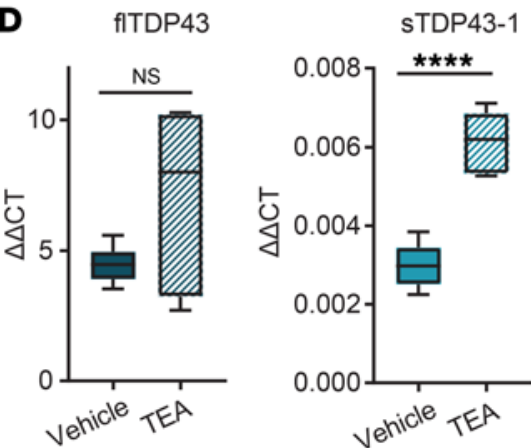

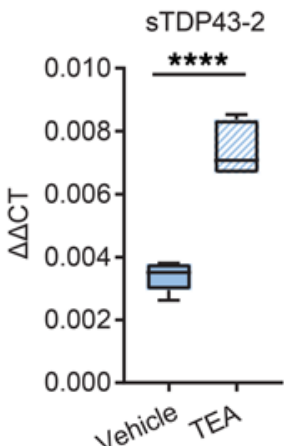

C
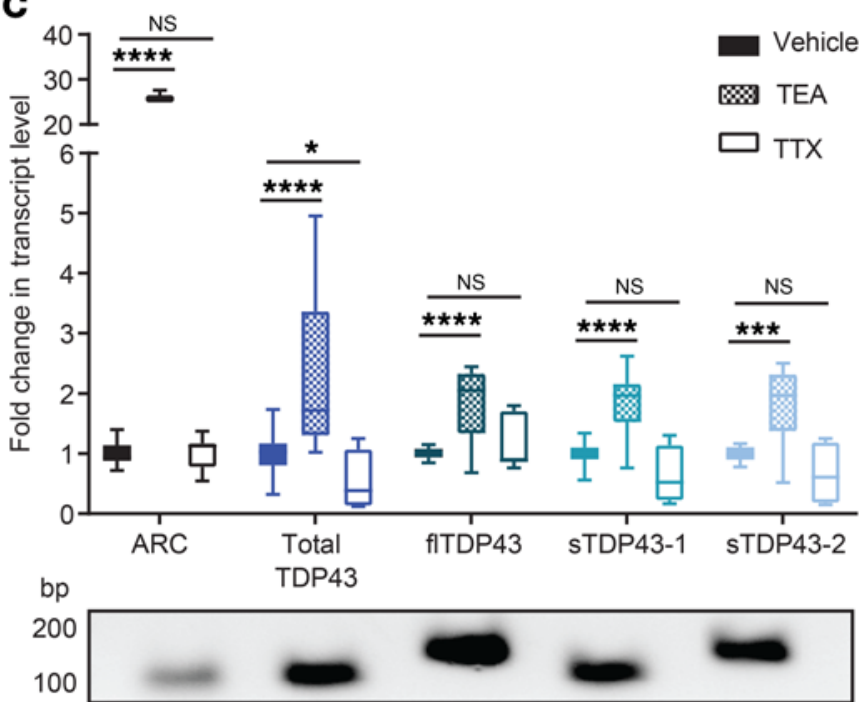

E

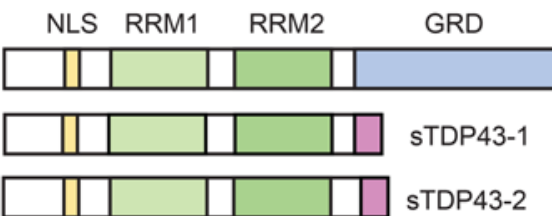

fITDP43

Figure 3. Hyperactivity drives alternative splicing of TARDBP. (A) Sashimi plot depicting splicing events for the TARDBP gene, assembled from HEK293T cell RNA-seq data (59). Splicing events predicted to skip the majority of exon 6 - encoding the TDP43 C-terminus - are highlighted in black. (B) Schematic of transcripts predicted to result in full-length TDP43 (fITDP43) and C-terminally shortened TDP43 (sTDP43). Green triangles indicate start codons, red triangles indicate stop codons, and PCR primers are color coded. (C) qRT-PCR of human iNeurons treated with TEA or TTX, showing activity-dependent upregulation of total and STDP43 or downregulation of sTDP43, respectively. ARC (activity-related cytoskeleton-associated protein) acts as a positive control for activity-dependent gene regulation. PCR products corresponding to each primer pair are shown below. Data combined from 3 replicates. ${ }^{*} P<0.05$, ${ }^{* * *} P<0.001$, ${ }^{* * *} P<0.0001$ by 1-way ANOVA with Dunnett's post hoc test. (D) Raw $\Delta \Delta C T$ values demonstrate the relatively low abundance of sTDP43-1 and -2 transcripts in iNeurons compared with fITDP43. (E) Schematic comparing fITDP43 and STDP43 proteins. The new sTDP43 C-terminus is shown in purple; NLS, nuclear localization signal; RRM, RNA-recognition motif; GRD, glycine-rich domain.

lesser mammals. Despite this, and the previous identification of sTDP43 splice variants in human and murine tissues $(60,61,64$, $65)$, the pathways governing their expression remain unknown. Our results demonstrate that these variants are dynamically and bidirectionally regulated by neuronal activity, with neuronal hyperactivity resulting in a significant upregulation of sTDP43 at the RNA and protein levels.

sTDP43 is cytoplasmically localized due to a putative NES in its C-terminal tail. To investigate sTDP43 localization, we transfected rodent primary mixed cortical neurons with diffusely localized mApple to enable visualization of neuronal cell bodies and processes, as well as constructs encoding flTDP43 or sTDP43-1 isoforms fused to an EGFP tag. We then imaged cultures by fluorescence microscopy to examine the localization of each isoform. flTDP43 appeared to be primarily nuclear in distribution, as expected, but sTDP43 demonstrated prominent cytoplasmic deposition (Figure 4A). The dramatic difference in sTDP43 localization was unanticipated given the presence of an intact nuclear localization signal (NLS) within the STDP43 N-terminus (Figure $3 \mathrm{E}$ ), and hinted at the presence of a potential nuclear export signal (NES) within the new sTDP43 C-terminus.
To explore this possibility, we utilized NetNES1.1, an algorithm that employs neural networks and hidden Markov models to predict NES-like motifs from protein primary structures (66). This analysis uncovered a series of amino acids near the STDP43 C-terminus that could potentially act as an NES (Figure 4B). We then tested the function of this putative NES through 3 complementary experiments. First, we altered the putative NES within sTDP 43 by site-directed mutagenesis (TSLKV $\rightarrow$ GGGGG) and expressed this construct [sTDP43(mNES)] in rodent primary neurons (Figure 4A). Protein localization was assessed by automated microscopy, using scripts that measure fluorescence separately within cytoplasmic and nuclear ROIs and calculate a nuclear/cytoplasmic ratio for TDP43 in each transfected neuron $(14,15)$. While sTDP 43 was localized to both the nucleus and cytoplasm, sTDP43(mNES) displayed a primarily nuclear distribution, more so even than flTDP43, suggesting that the putative NES is necessary for cytoplasmic deposition of sTDP43 (Figure 4C). Second, we fused EGFP to the 18-amino acid tail of sTDP43 (EGFP-tail), or a version of the sTDP43 tail harboring a mutated NES [EGFP-tail(mNES)] (Figure 4D). For comparison, we also expressed Shuttle-RFP, a construct with a strong NES and a weak NLS that exhibits a pre- 
A
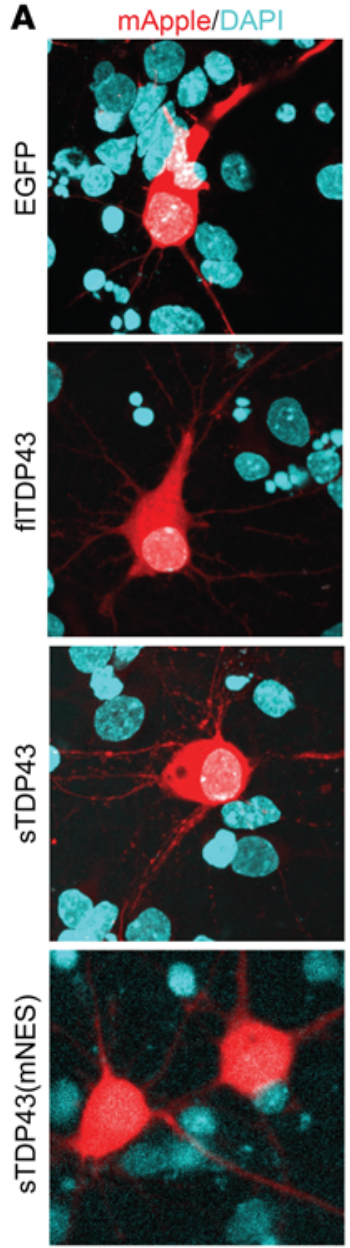

EGFP-tag
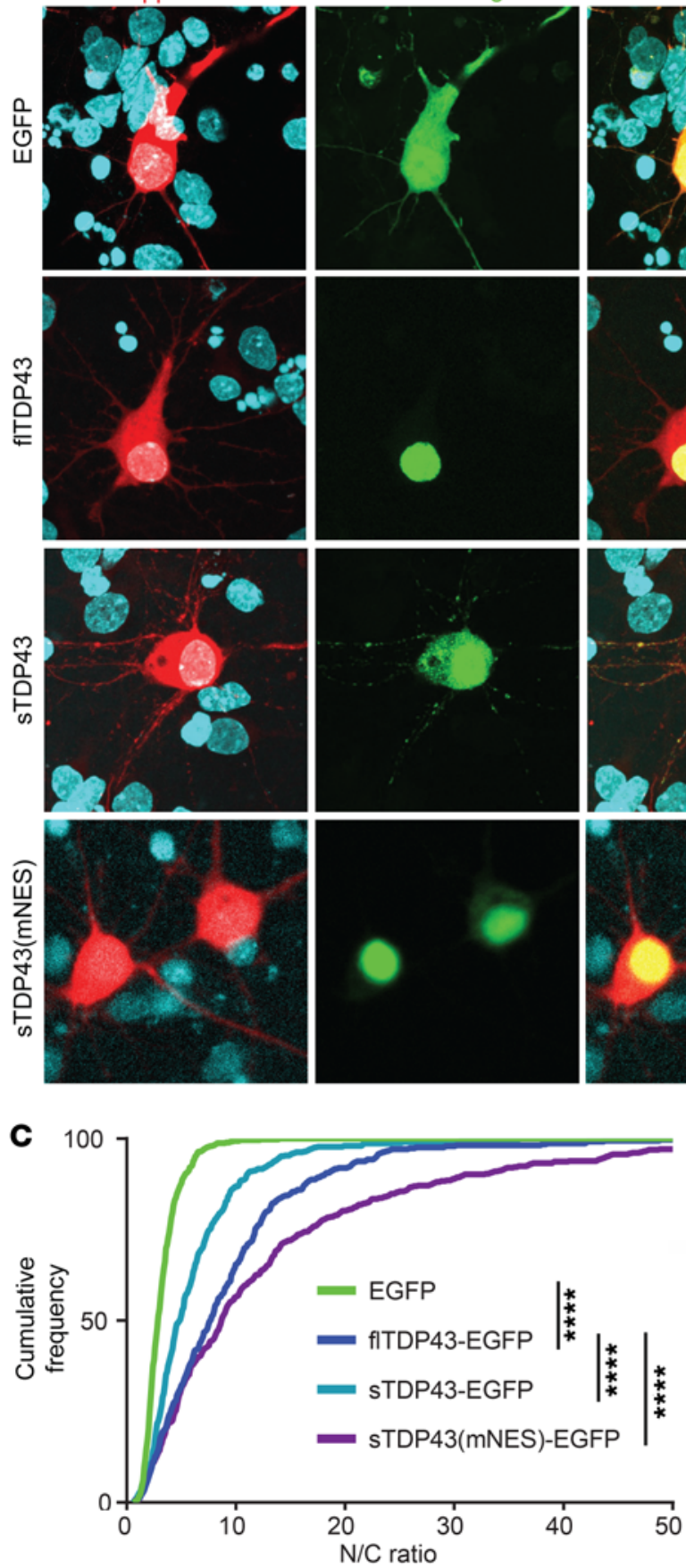

B
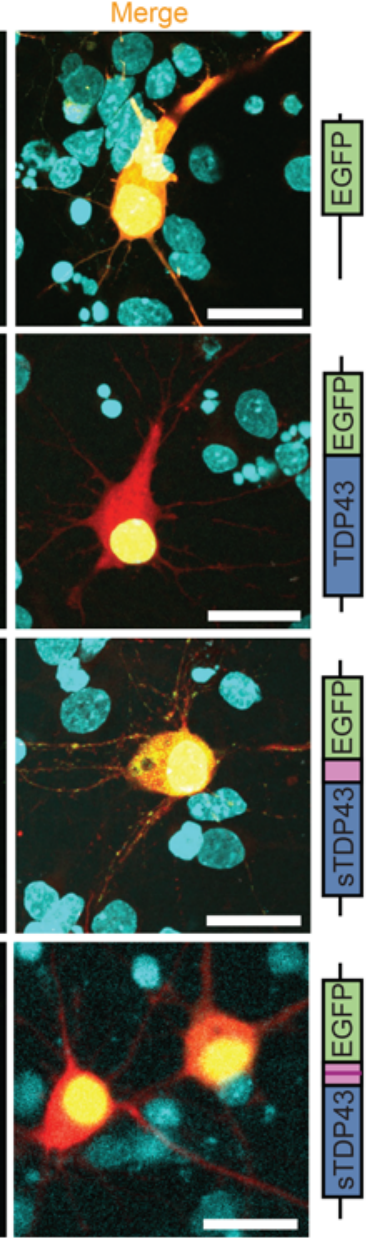

D

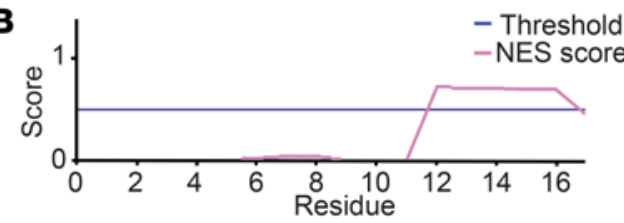

VHL I SNVYGRSTSLKVVL

Putative NES
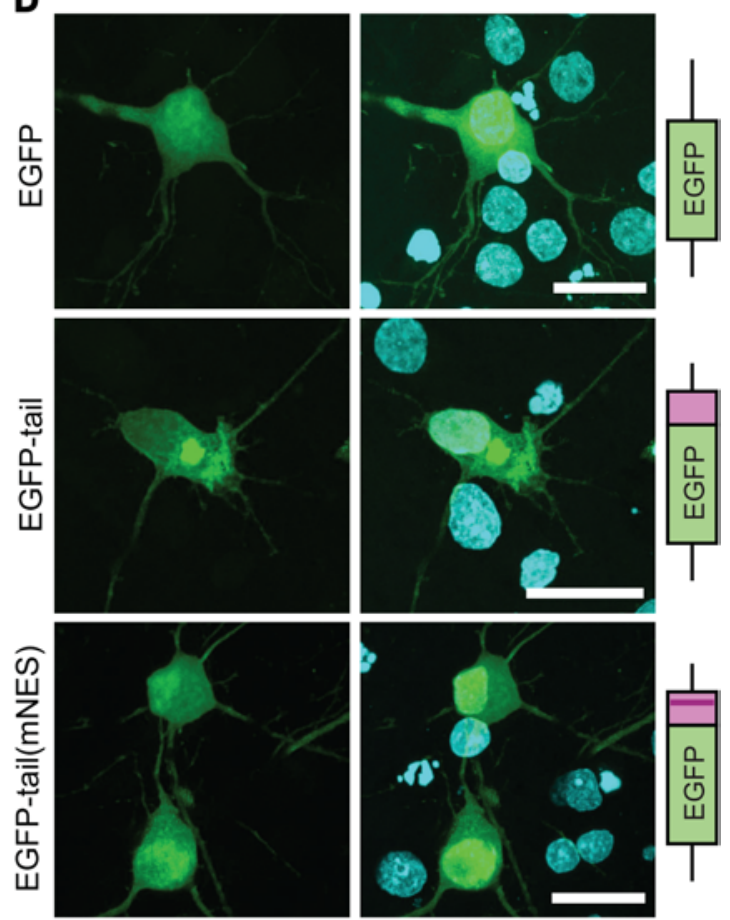

Figure 4. sTDP43 accumulates within the cytoplasm due to a putative NES. (A) Rodent primary mixed cortical neurons were transfected with mApple and EGFP-tagged TDP43 isoforms, and then imaged by fluorescence microscopy. (B) Amino acid sequence of the sTDP43 tail showing the putative NES identified using NetNES 1.1. Light blue, polar; purple, positively charged; green, hydrophobic residues. (C) sTDP43-EGFP was significantly more cytoplasmic compared with fITDP43-EGFP, while mutation of the putative NES (mNES) restores nuclear localization. N/C, nuclear/cytoplasmic. EGFP $n=481$, fITDP43-EGFP $n=385$, sTDP43-EGFP $n=456$, sTDP43(mNES)-EGFP $n=490$, stratified among 3 replicates. ${ }^{* * *} P<0.0001$ by 1 -way ANOVA with Dunnett's post hoc test. (D) Rodent primary mixed cortical neurons were transfected with 1 of 3 constructs: EGFP alone, EGFP fused to the sTDP43 C-terminus, or EGFP fused to the sTDP43 C-terminal tail harboring a mutated NES (mNES). (E) The sTDP43 C-terminus mislocalizes EGFP to the cytoplasm, and mislocalization depends on the putative NES. Shuttle-RFP, a construct with a strong NES, serves as a positive control for a cytoplasmic protein. EGFP $n=2490$, Shuttle-RFP $n=2073$, EGFP-tail $n=$ 1956, EGFP-tail(mNES) $n=2482$, combined from 3 replicates. ${ }^{* * *} P<0.0001$ by 1 -way ANOVA with Dunnett's post hoc test. Scale bars: $20 \mu \mathrm{m}$ (A and D).

dominantly cytoplasmic distribution (67). Addition of the sTDP43 tail was sufficient to partially exclude EGFP-tail from the nucleus, but this change in distribution was eliminated by mutating the residues making up the putative NES in EGFP-tail(mNES) (Figure
4E). Lastly, we asked whether sTDP43's cytoplasmic distribution arises from the absence of a nuclear retention signal encoded within the canonical TDP43 C-terminus (68), or the presence of an active NES within the sTDP43 tail. Fusing the sTDP43 tail to 
A
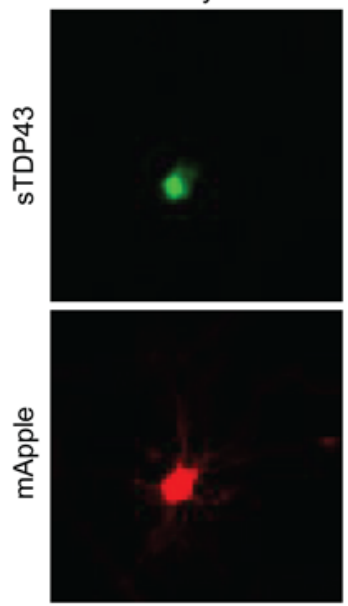

Day 2
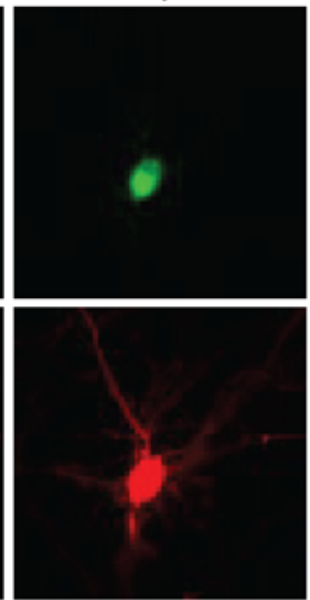

Day 3
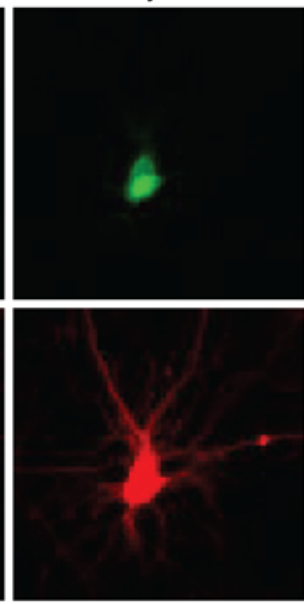

Day 4
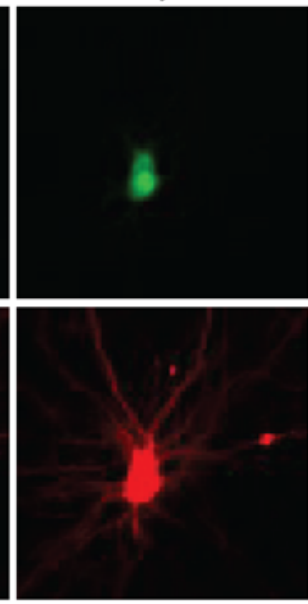

Day 5

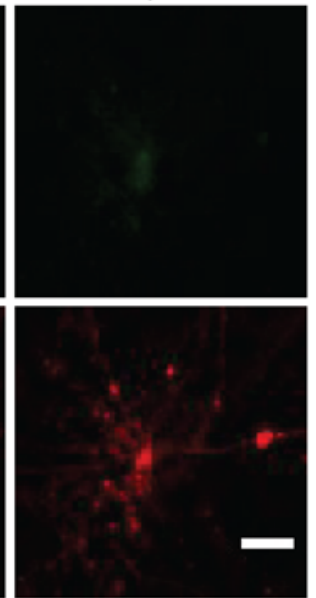

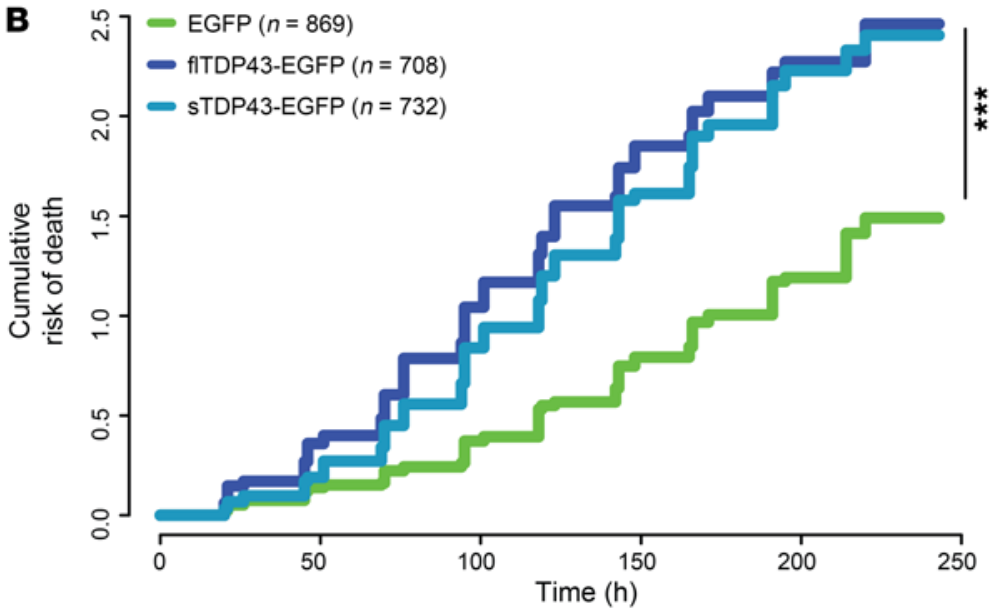

Figure 5. sTDP43 overexpression is neurotoxic. (A) Example of a single neuron expressing mApple and sTDP43-EGFP, tracked by longitudinal fluorescence microscopy. Fragmentation of the cell body and loss of fluorescence indicates cell death. (B) The risk of death was significantly greater in neurons overexpressing sTDP43-EGFP and fITDP43-EGFP, compared with those expressing EGFP alone. EGFP $n=869$, fITDP43-EGFP $n=708$, sTDP43-EGFP $n=732$, stratified among 3 replicates. ${ }^{* *} P<2 \times 10^{-16}$ by Cox proportional hazards analysis. Scale bar: $20 \mu \mathrm{m}$ (A)
flTDP43 markedly shifted the distribution of flTDP43 to the cytoplasm (Supplemental Figure 3), suggesting that STDP43 localization is dictated primarily by the C-terminal NES, and not the lack of a nuclear retention signal. Together, these data indicate that the distinct sTDP43 C-terminus encodes a functional NES that facilitates cytoplasmic accumulation of STDP43.

sTDP43 overexpression is neurotoxic. TDP43 mislocalization is a widely observed phenomenon in ALS, and cytoplasmic TDP43 is a strong predictor of cell death (14). Given these data and the largely cytoplasmic localization of sTDP43, we surmised that sTDP43 accumulation would be toxic to mammalian neurons. We therefore utilized automated microscopy in conjunction with survival analysis to track individual neurons prospectively over time and determine their risk of death in an unbiased and high-throughput manner $(14,15,59,69,70)$. Rodent primary mixed cortical neurons were transfected with mApple and EGFP-tagged TDP43 isoforms and imaged by fluorescence microscopy at 24-hour intervals for 10 days (71). Custom scripts were used to automatically generate ROIs corresponding to each cell and determine time of death based on rounding of the soma, retraction of neurites, or loss of fluorescence (Figure $5 \mathrm{~A}$ ). The time of death for individual neurons was used to calculate the risk of death in each population relative to a reference group, in this case neurons expressing $\operatorname{EGFP}(71,72)$. In keeping with the results of previous studies, flTDP43 overexpression resulted in a significant increase in the risk of death in comparison with EGFP alone $\left(P<2 \times 10^{-16}\right)$. sTDP43-1 overexpression elicited an analogous increase in the risk of death for transfected neurons $(P<$ $\left.2 \times 10^{-16}\right)$, suggesting that sTDP43 and flTDP43 display similar toxicity when overexpressed in neurons (Figure $5 \mathrm{~B}$ ).

sTDP43 alters endogenous TDP43 localization. TDP43 dimerizes via its N-terminus $(52,73-79)$, and because sTDP43 is exported from the nucleus and contains an intact $\mathrm{N}$-terminus we questioned whether sTDP43 might bind to and sequester endogenous flTDP43 within the cytoplasm. To determine if sTDP43 is capable of interacting with endogenous flTDP43, we transfected HEK293T cells with HaloTag-labeled sTDP43-1 or flTDP43 and isolated the fusion proteins using HaloLink resin (Figure 6A). We detected equivalent amounts of endogenous flTDP43 in eluates from sTDP43-HaloTag and flTDP43-HaloTag, indicating that sTDP43 effectively binds endogenous flTDP43 (Figure 6B).

We also examined the interaction between sTDP43 and endogenous TDP43 by ICC. HEK293T cells were transfected with EGFP or EGFP-tagged sTDP43, immunostained using a C-terminal TDP43 antibody that recognizes endogenous TDP43 but not sTDP43, and imaged by confocal fluorescence microscopy (Fig- 
$\mathbf{A}$
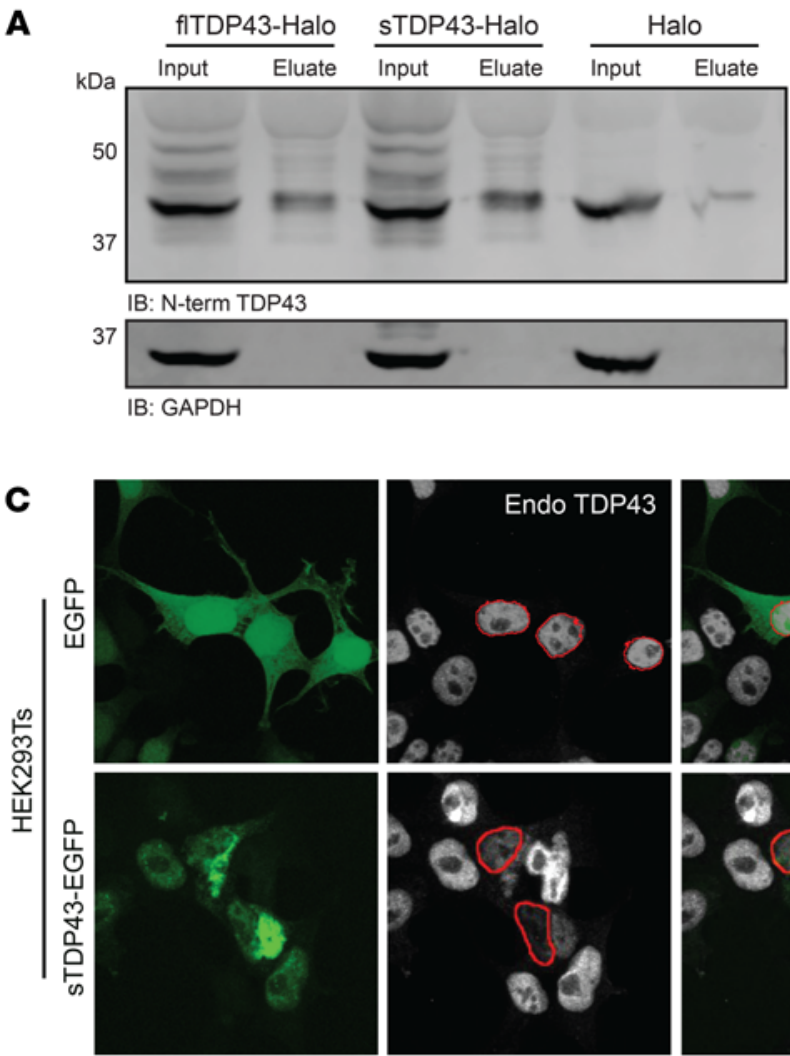

$\mathbf{F}$
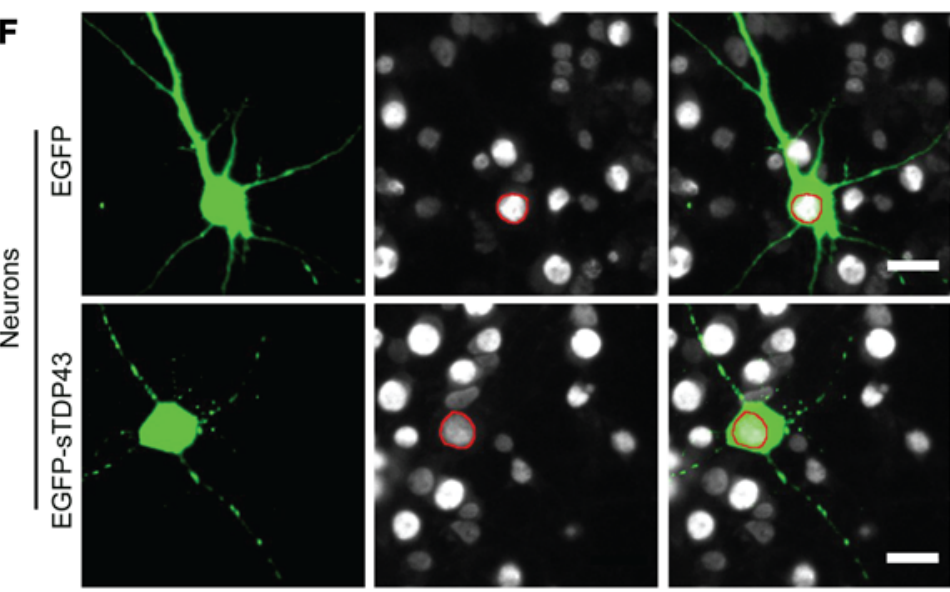

B

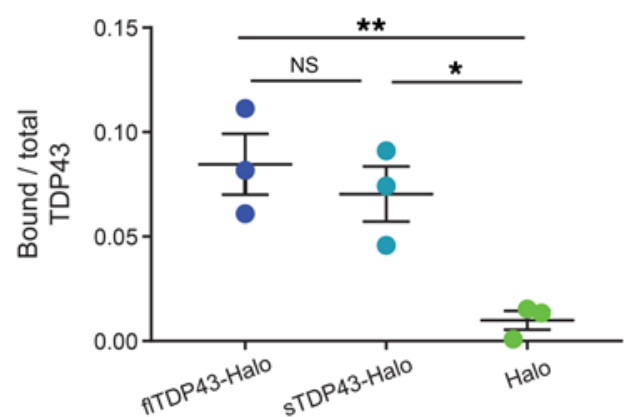

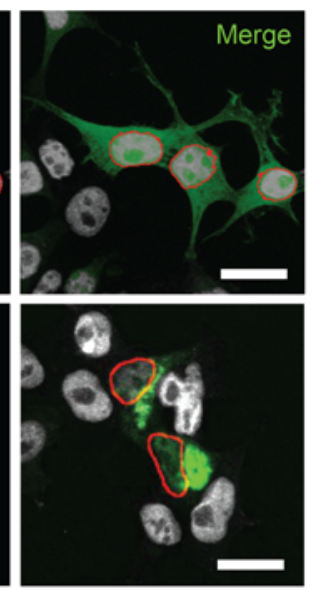

D

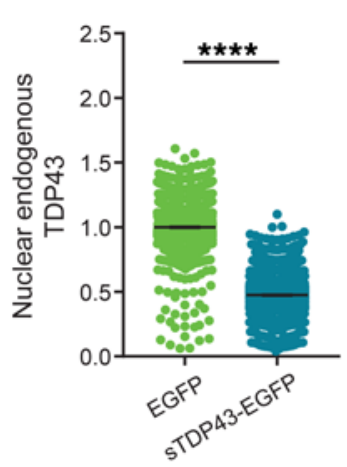

G

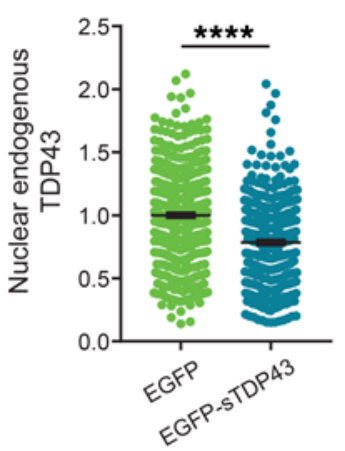

$\mathbf{E}$

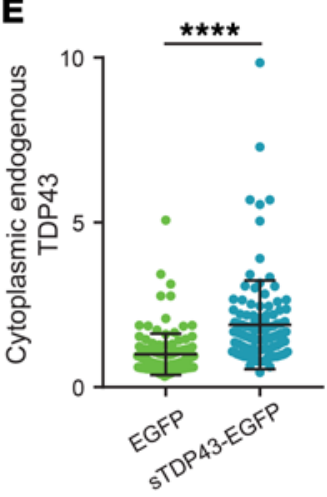

H

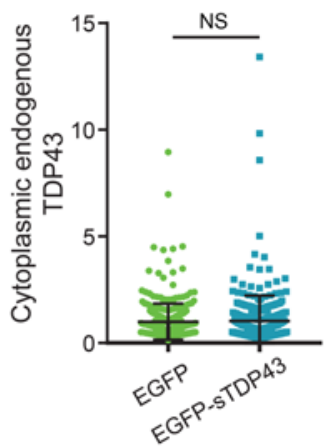

Figure 6. sTDP43 overexpression leads to the cytoplasmic deposition and nuclear clearance of endogenous TDP43. (A) HaloTag fusions of fITDP43 or STDP43 were expressed in HEK293T cells and immunoprecipitated with HaloLink. Bound TDP43 was immunoblotted (IB) with a C-terminal TDP43 antibody. GAPDH served as a loading control. (B) Quantification of data shown in A, demonstrating the fraction of total TDP43 bound to fITDP43-Halo, sTDP43-Halo, or Halo alone. Data were combined from 3 replicates. ${ }^{*} P<0.05,{ }^{*} P<0.01$ by 1 -way ANOVA with Dunnett's post hoc test. (C) HEK293T cells were transfected with EGFP or EGFP-tagged sTDP43, and then immunostained using an antibody that recognizes the endogenous TDP43 C-terminus (Endo). Red, nuclear regions of interest (ROIs) determined by DAPI staining. (D) Nuclear, endogenous TDP43 is reduced by sTDP43 overexpression in HEK293T cells. EGFP $n=1537$, sTDP43-EGFP $n=1997,3$ replicates. ${ }^{* * *} P<0.0001$ by 2 -tailed $t$ test. (E) Cytoplasmic endogenous TDP43 is elevated by sTDP43 overexpression in HEK293T cells. ECFP $n=129$, sTDP43-EGFP $n=113,3$ replicates. ${ }^{* * *} P<0.0001$ by 2 -tailed $t$ test. (F) Primary mixed rodent cortical neurons were transfected with EGFP or EGFP-tagged sTDP43, and then immunostained using a C-terminal TDP43 antibody. Red, nuclear ROIs determined by DAPI staining. (G) STDP43 overexpression resulted in a significant drop in nuclear, endogenous TDP43 in primary neurons (EGFP $n=395$, EGFP-sTDP43 $n=323,3$ replicates; ${ }^{* * *} P<0.0001$ by 2 -tailed $t$ test), but this was not accompanied by increases in cytoplasmic, endogenous TDP43 (H) (EGFP $n=394$, EGFP-sTDP43 $n=323,3$ replicates, NS by 2 -tailed $t$ test). Scale bars: $20 \mu \mathrm{m}$ (C and $\mathbf{F}$ ).

ure 6C). HEK293T cells overexpressing EGFP-tagged sTDP43 displayed cytoplasmic inclusions that strongly colocalize with endogenous TDP43. Moreover, we observed significant reductions in nuclear endogenous TDP43 in association with cytoplas- mic TDP43 deposition (Figure 6, D and E), suggesting cytoplasmic sequestration of endogenous TDP43 by sTDP43. Rodent primary mixed cortical neurons overexpressing sTDP43-1 displayed a similar depletion of endogenous TDP43 from the nucleus (Figure 6, F 
A Mouse Frontal Cortex

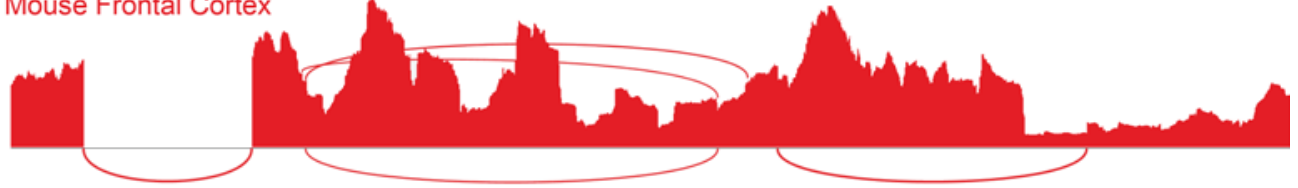

Mouse Lumbar Motor Neurons

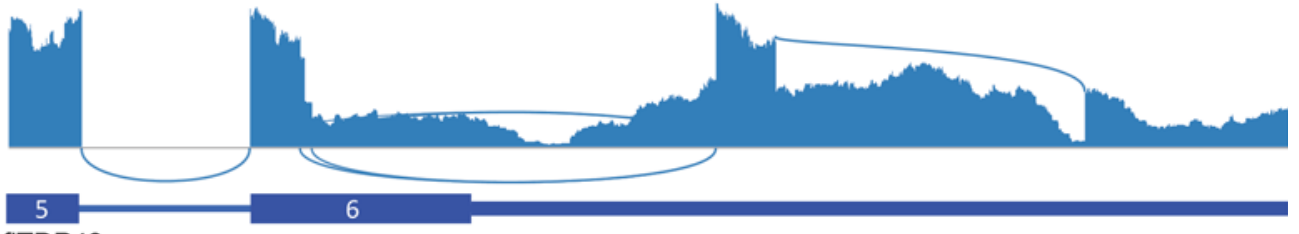

fITDP43

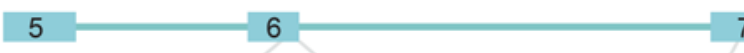

STDP43-1 ERS GRFG

VHLISNVYGRSTSLKVVL *

STDP43-2 ERSGRFGGNP

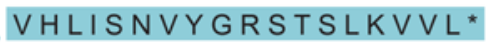

B
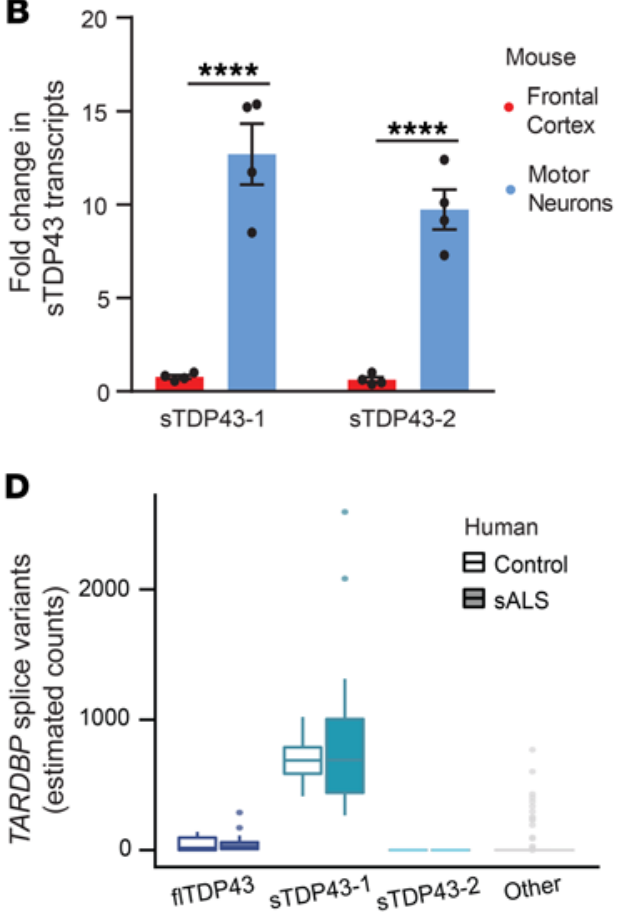
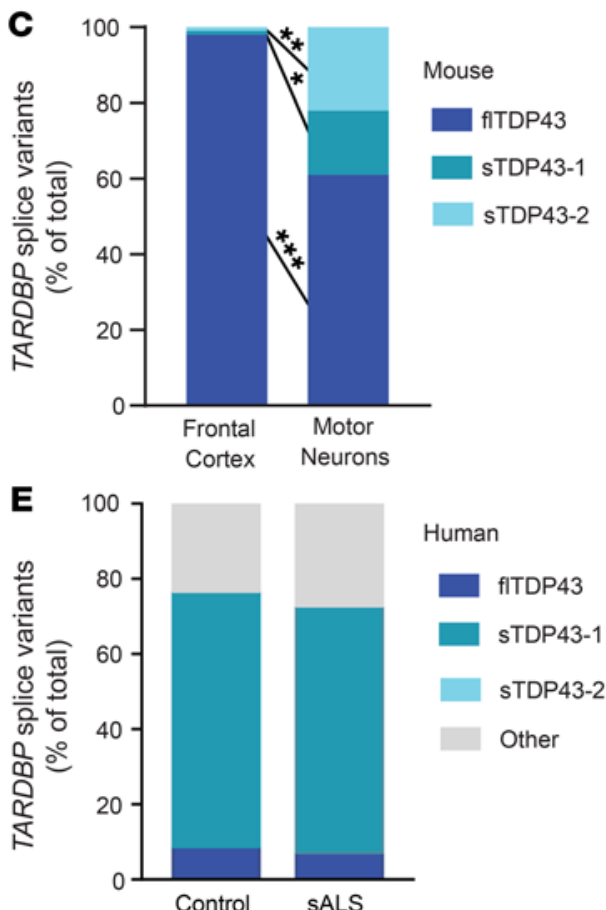

Figure 7. sTDP43 transcripts are enriched in lumbar motor neurons. (A) Sashimi plots depicting TARDBP splicing in murine frontal cortex homogenate (red) or microdissected lumbar motor neurons (blue). Numbers correspond to exons, and asterisks represent stop codons. (B) Both sTDP43-1 and sTDP43-2 splice events are highly enriched in lumbar motor neurons compared with frontal cortex homogenate. Graph depicts read counts normalized to reads per million for each library. Analysis includes 4 replicates. ${ }^{* * *} P<0.0001$ by multiple $t$ test with the Holm-Sidak correction. (C) While sTDP43-1 and -2 each comprise approximately $1 \%$ of the total TARDBP transcripts in frontal cortex homogenate, they make up $17 \%$ and $22 \%$ of total TARDBP transcripts in lumbar motor neurons, respectively. Frontal cortex $n=6$, lumbar motor neurons $n$ $=4 .{ }^{*} P<0.05,{ }^{* *} P<0.01,{ }^{* * *} P$ $<0.001$ by 2-way ANOVA with Sidak's multiple-comparisons test. (D) sTDP43-1 is enriched within lumbar motor neurons microdissected from both control $(n=9)$ and SALS $(n=13)$ patient tissue. (E) sTDP43-1 makes up the majority of total TARDBP transcripts in both control and sALS patient lumbar motor neurons. and G). Thus, sTDP43 overexpression results in both cytoplasmic deposition and nuclear clearance of endogenous TDP43, recapitulating signature features of ALS pathology and implying that both gain- and loss-of-function mechanisms contribute to toxicity.

In sTDP43-transfected cells, we observed significant variability in the degree of TDP43 nuclear exclusion and cytoplasmic aggregation, which we suspected was due to differences in STDP43 expression among transfected cells. Because the abundance of a fluorescently labeled protein is directly proportional to the intensity of the fluorescent tag (80), we estimated sTDP43 expression in each cell by measuring single-cell EGFP intensity, and separated cells into 5 bins based on sTDP43 expression level. In doing so, we observed a direct relationship between the extent of endogenous TDP43 mislocalization and sTDP43 expression (Supplemental Figure 4). These results indicate that TDP43 pathology may become more prevalent as sTDP43 expression is upregulated by neuronal hyperexcitability or other mechanisms.

We failed to observe significant increases in cytoplasmic TDP43 deposition in transfected primary neurons (Figure $6 \mathrm{H}$ ), potentially due to steric inhibition of sTDP43 localization and function by fusion with EGFP or HaloTag (Supplemental Figure 5). Placing the EGFP tag on the C-terminus of sTDP43 partially prevented cytoplasmic localization of sTDP43-EGFP (Supplemental Figure 5, A-C), likely by masking the C-terminal NES. Similarly, we found that fusion of HaloTag with the N-terminus of sTDP43 significantly inhibits its binding to endogenous TDP43 (Supplemental Figure 5, D and E). As such, N-terminal labeling of sTDP43 leaves the NES accessible but blocks association with endogenous TDP43, while C-terminal sTDP43 labeling obstructs the NES but allows interaction with endogenous TDP43. 
sTDP43 lacks canonical functions of flTDP43. To further examine the possibility that STDP43 elicits loss-of-function toxicity, we assessed the ability of STDP43 to participate in TDP43-related splicing activity. In keeping with TDP43's function as a splicing repressor, TDP43 effectively blocks the inclusion of cystic fibrosis transmembrane conductance regulator (CFTR) exon $9(81,82)$ (Supplemental Figure 6A). In HEK293T cells expressing a CFTR minigene reporter, cotransfection with EGFP-flTDP43 resulted in proficient exon 9 exclusion as measured by PCR. In contrast, EGFP-sTDP43-1 expression failed to significantly affect exon 9 exclusion (Supplemental Figure 6, B and C), suggesting that without the C-terminus, sTDP43 is incapable of TDP43-specific splicing $(60,64,65)$.

Functional flTDP43 also participates in an autoregulatory feedback loop, in which flTDP43 recognizes sequences within the TARDBP 3'UTR, triggering nuclear retention and/or alternative splicing and subsequent mRNA degradation $(83,84)$. To determine if sTDP43 is able to regulate endogenous TDP43 expression via this mechanism, we employed a TDP43 autoregulation reporter consisting of an open reading frame $(\mathrm{ORF})$ encoding the fluorescent protein mCherry upstream of the TARDBP 3'UTR (69) (Supplemental Figure 7A). In rodent primary cortical neurons expressing the TDP43 autoregulation reporter, cotransfection with EGFP-tagged flTDP43 resulted in a decrease in reporter signal, as expected. EGFP-labeled sTDP43-1 had more subtle effects on reporter fluorescence, suggesting that its ability to autoregulate TDP43 is impaired (Supplemental Figure 7, B and C). Likewise, when expressed in HEK293T cells, sTDP43-1 exhibited a similarly muted effect on endogenous TDP43 at the transcript and protein level (Supplemental Figure 7, D-F), consistent with poor autoregulation. Together, these results indicate that STDP43 lacks many of the canonical functions of TDP43, including its splicing and autoregulatory abilities.

sTDP43 colocalizes with markers of stress granules. Previous studies suggested that STDP43 associates with protein components of cytoplasmic stress granules, including G3BP1 and TIA1 (65). Therefore, we immunostained for G3BP1 and TIA1 in HEK293T cells overexpressing EGFP-tagged sTDP43-1, before and after application of osmotic stress (0.4 M sorbitol). Prior to sorbitol treatment, we noted substantial colocalization of sTDP431 with G3BP1 (Supplemental Figure 8A) and TIA1 (data not shown) in large cytoplasmic deposits; these structures were unique to cells transfected with sTDP43-1, suggesting that sTDP43 overexpression elicits the formation of irregular structures rich in stress granule components. However, when cells were stressed with 0.4 M sorbitol we observed the formation of multiple small, punctate structures resembling stress granules that colocalize with both G3BP1 and TIA1, as well as endogenous TDP43 (Supplemental Figure 8B). Moreover, while osmotic stress drives flTDP43 to the cytoplasm, it has little effect on sTDP43-1 localization (Supplemental Figure 8C). These data confirm that sTDP43 localizes to stress granules, and further imply that sTDP43 production may be sufficient for the assembly of cytoplasmic stress granule-like structures even in the absence of stress.

sTDP43 transcripts are enriched in murine and human lumbar motor neurons. To determine if STDP43 isoforms are produced in vivo and assess their expression in different regions of the CNS, we took advantage of a previous study that analyzed the transcriptome from murine frontal cortex and lumbar spinal motor neurons isolated by laser capture microdissection (Figure 7A) (85). The most abundant splice isoform in frontal cortex homogenate was flTDP43, with predominant use of the conventional termination codon within TARDBP exon 6. However, splicing of the TARDBP locus, and in particular exon 6 and the $3^{\prime} \mathrm{UTR}$, was dramatically altered in murine spinal motor neurons. In contrast with what was observed in frontal cortex, 2 splicing events corresponding to sTDP43 variants 1 and 2 were strongly favored in spinal motor neurons - these isoforms were upregulated approximately 12and 10-fold, respectively, in lumbar spinal neurons relative to frontal cortex (Figure 7, B and C). Further, while an ALS-associated TARDBP mutation (Q331K) did not affect sTDP43 transcript abundance, we noted age-related increases in STDP43 mRNA levels in 20-month- versus 5-month-old mouse cortices (Supplemental Figure 9). These data show that sTDP43 isoforms are not only detectable in vivo within the murine CNS, but they are also significantly upregulated by age and enriched in spinal motor neurons, a cell type selectively targeted in ALS.

We also examined sTDP43 expression in human spinal neurons utilizing published RNA-seq data from laser-captured lumbar spinal motor neurons, isolated from control and sALS patient tissue (86). Within this data set, we identified specific transcripts corresponding to flTDP43, sTDP43-1, and sTDP43-2, and characterized the remaining TARDBP variants as "other." Although there was no apparent difference in the abundance of any TARDBP transcripts between sALS and control motor neurons, we noted a dramatic enrichment of sTDP43-1 transcripts in human spinal neurons, in comparison with flTDP43, sTDP43-2, and other TARDBP variants (Figure 7, D and E). Furthermore, and in contrast with what we observed in rodent spinal neurons, sTDP43-1 was the predominant TARDBP splice isoform detected in human spinal neurons. To extend these findings, we also examined available RNA-seq data from spinal cord ventral horn homogenate from control and sALS patients (87), as well as cerebellum and frontal cortex from controls, individuals with sALS, and patients bearing disease-associated mutations in C9ORF72 (C9ALS) (88). Despite fundamental differences in sample preparation and sequencing methodology that prevented the direct comparison of transcript abundance between tissue types (Supplemental Figure 10A), we consistently observed significant expression of sTDP43-1 but not sTDP43-2 in several different regions of the CNS, including but not limited to spinal motor neurons, cerebellum, and frontal cortex, though there were no differences between control, sALS, and C9ALS patient samples (Supplemental Figure 10, B and C).

Endogenously produced sTDP43 is detectable using specific antibodies. To distinguish natively produced sTDP43 species, we generated an antibody directed against the unique 18-amino acid C-terminus of sTDP43 (Figure $3 \mathrm{E}$ ). This antibody specifically recognized the sTDP43 C-terminus fused to EGFP, suggesting that the sTDP43 tail is sufficient for immunoreactivity, and the signal was completely abolished by preincubation with the immunizing peptide (Supplemental Figure 11A). Furthermore, expression of artificial miRNAs (amiRNAs) targeting TDP43 $(69,89)$ effectively reduced flTDP43 levels, as expected (Supplemental Figure 11, B and C), and also decreased sTDP43 immunoreactivity (Supplemental Figure 11, D and E), confirming antibody specificity. We further validated the sTDP43 antibody by transfecting HEK293T 
A
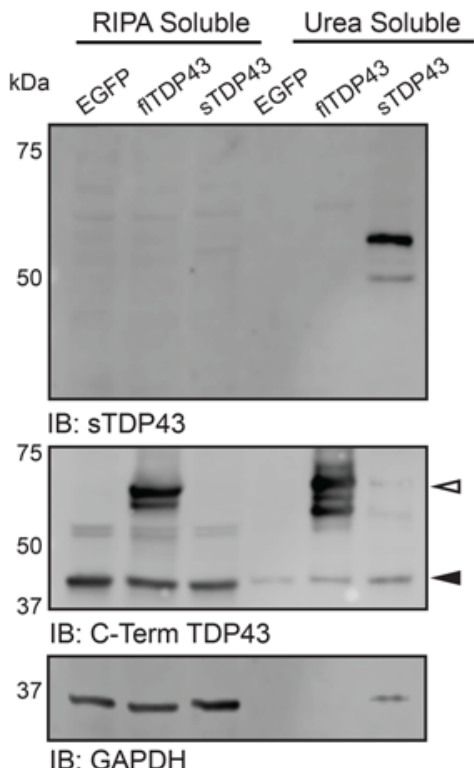

B
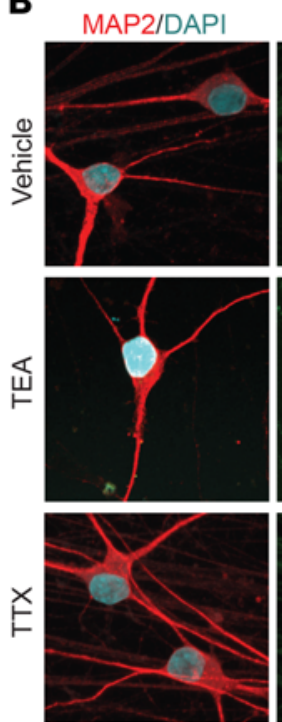

sTDP43
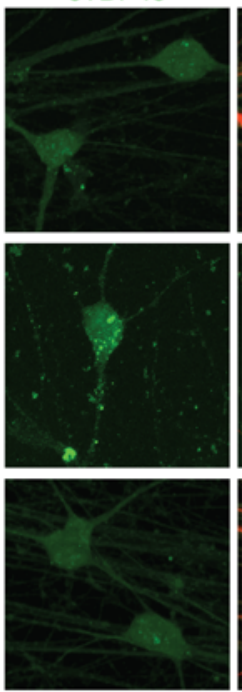

Merge
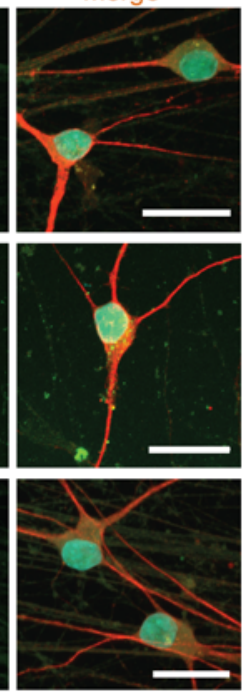

C

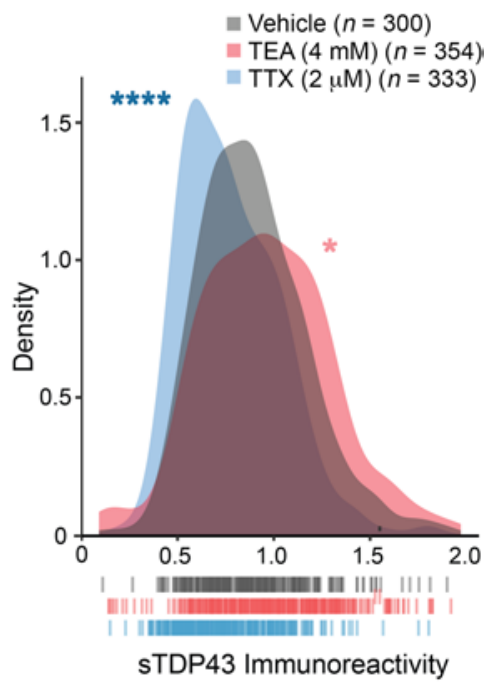

D
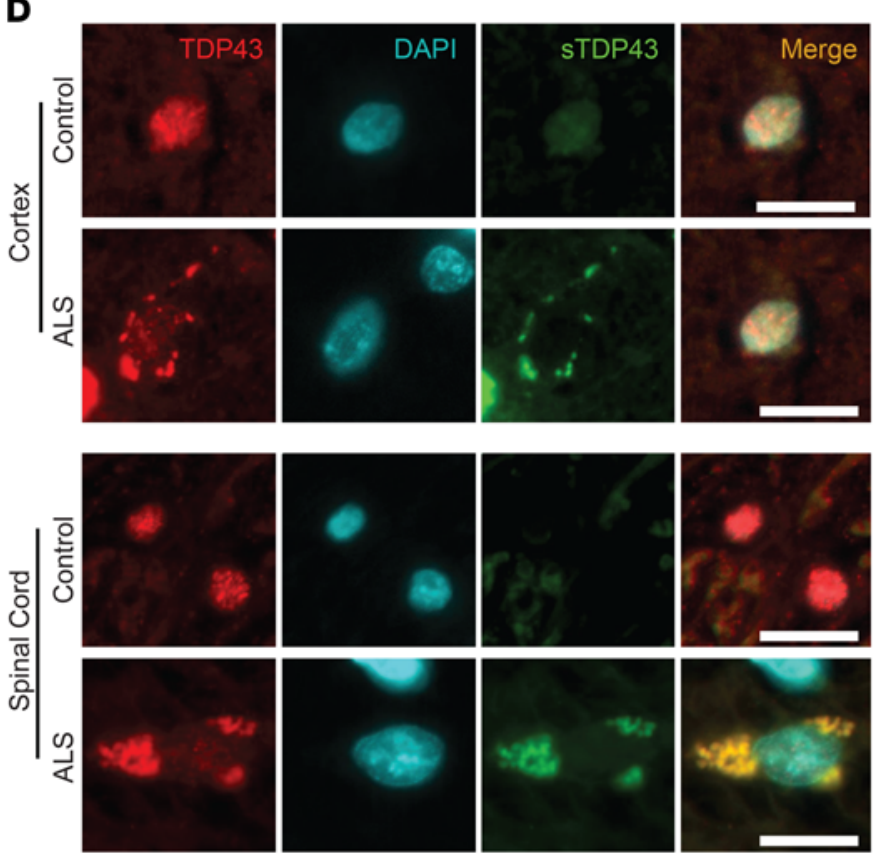

$\mathbf{F}$
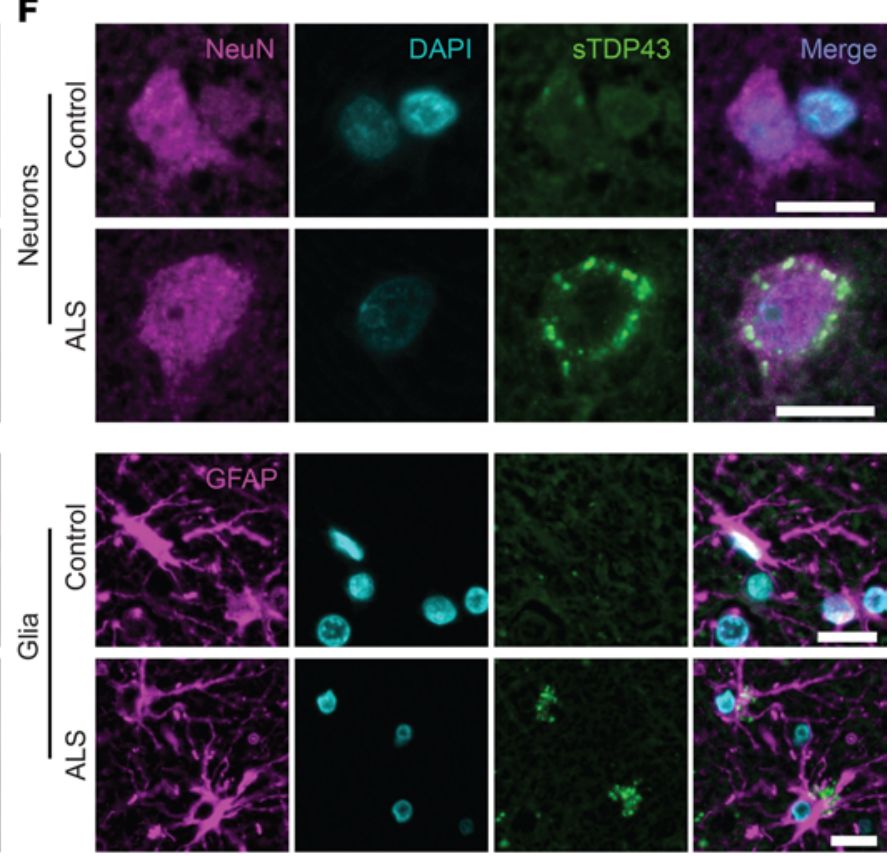

E

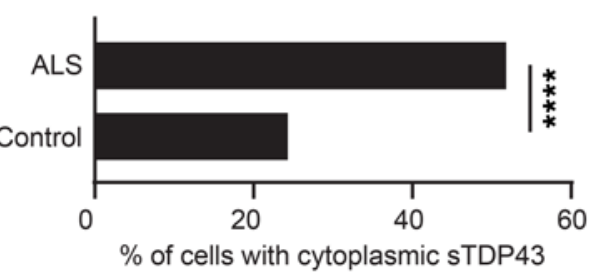

Figure 8. Endogenous sTDP43 is detectable in vivo by antibodies generated against its distinct C-terminus. (A) Western blot of EGFP-tagged fITDP43 or sTDP43 overexpressed in HEK293T cells. Black arrowhead, endogenous TDP43; white arrowhead, EGFP-fITDP43. (B) ICC using sTDP43 antibodies showed increased immunoreactivity in TEA-treated iNeurons and decreased immunoreactivity in TTX-treated iNeurons. (C) Density plot depicting the change in STDP43 immunoreactivity between conditions. Vehicle $n=300$, TEA $n=354$, TTX $n=333$, 3 replicates; vertical lines indicate single neurons. ${ }^{*} P<0.05$, ${ }^{* * * *} P<0.0001$ by Kolmogorov-Smirnov test. (D) IHC comparing the distribution of N-terminal TDP43 and sTDP43 in spinal cord and cortex from patients with sporadic ALS and controls. (E) Quantification of cells with cytoplasmic STDP43 in control and ALS patient spinal cord (control $n=115$, ALS $n=110$; data representative of 2 control and 3 ALS patients). ${ }^{* * *} P<0.0001$ by Fisher's exact test. (F) IHC demonstrating neuronal and glial sTDP43 accumulation in cortex from individuals with sALS and controls. Scale bars: $20 \mu \mathrm{m}$ (B, D, and F). 
cells with EGFP-tagged sTDP43-1, isolating RIPA- and urea-soluble protein fractions, and immunoblotting for STDP43. In previous studies, overexpressed sTDP43 was highly insoluble (64); supporting this, we detected EGFP-sTDP43 exclusively in the urea-soluble fraction, while EGFP-flTDP43 appeared in both RIPA- and urea-soluble fractions (Figure 8A). We also tested the sTDP43 antibody in human iNeurons treated with TEA or TTX to induce or abolish neuronal activity, respectively (Figure 8B). In these studies, TEA increased sTDP43 immunoreactivity, while TTX reduced sTDP43 levels (Figure 8C), consistent with activity-dependent upregulation of N-terminally labeled D2-TDP43 (Figure 2) and its detection by antibodies specific for the TDP43 N-terminus (Figure 1). Notably, sTDP43 antibodies detected numerous cytoplasmic puncta in TEA-treated neurons that were less apparent in vehicle- and TTX-treated cells, and the background nuclear signal was minimal in all cases. Identical STDP43-positive cytoplasmic puncta were observed in rodent primary mixed cortical neurons treated with bicuculline (Supplemental Figure 11F). These data indicate that sTDP43 antibodies selectively detect truncated, cytoplasmic, and insoluble TDP43 species by Western blot and ICC, establishing them as useful tools for investigating sTDP43 deposition and its potential role in neurodegeneration.

Based on the observed upregulation of sTDP43 splice isoforms in lumbar spinal neurons, we employed our newly developed sTDP43 antibody for detecting sTDP43 in vivo within murine spinal cord sections. As predicted from the RNA-seq data, we detected cytoplasmic sTDP43 in anterior horn neurons from the lumbar spinal cord (Supplemental Figure 12A), confirming the subcellular distribution of the protein originally noted in vitro. We also observed strong colocalization of sTDP43 with glial fibrillary acidic protein-positive (GFAP-positive) astrocytic projections within the spinal white matter, indicating astrocytic expression of sTDP43 (Supplemental Figure 12B). Subsequent studies confirmed that sTDP43 is endogenously produced by human iPSC-derived astrocytes (Supplemental Figure 13), suggesting that while STDP43 is enriched within spinal neurons (Figure 7), it is also synthesized by supporting glia.

sTDP43 pathology is observed in ALS patient tissue. Given that (a) STDP43 is endogenously produced at relatively high levels in spinal motor neurons, (b) neuronal hyperexcitability is a conserved feature of ALS, and (c) sTDP43 is upregulated by neuronal activity and age, we suspected that STDP43 may accumulate in individuals with sALS. To address this question, we immunostained human cortex and spinal cord sections from sALS, C9ALS, and unaffected control patients using antibodies that recognize the TDP43 $\mathrm{N}$-terminus or our newly developed sTDP43 antibodies (Figure 8D). As predicted, immunostaining with N-terminal TDP43 antibodies showed both a reduction in nuclear signal and the appearance of cytoplasmic inclusions selectively in ALS patient tissue. Although control tissue exhibited low immunoreactivity for STDP 43 in both the cortex and spinal cord, we observed a striking accumulation of sTDP43 within cytoplasmic deposits in ALS spinal cord and cortex (Figure 8E). sTDP43-positive inclusions closely colocalized with N-terminally reactive cytoplasmic aggregates but not residual nuclear TDP43, suggesting that sTDP43 antibodies specifically label cytoplasmic deposits in ALS tissue. In this limited case study, sTDP43 pathology appeared to be conserved between sALS and C9ALS (Supplemental
Figure 14A), hinting at a conserved process. We also observed a tight correlation between conventional TDP43 pathology (nuclear exclusion and cytoplasmic aggregation) and STDP43 deposition - in ALS samples, neurons displaying TDP43 nuclear exclusion almost always showed cytoplasmic sTDP43 pathology. Additionally, we noted several cells from ALS patients that exhibited sTDP43 deposits despite a normal nuclear TDP43 pattern, perhaps illustrating an early stage of pathology (Supplemental Figure 14, B and C). Even so, we detected significant heterogeneity in STDP43 pathology among ALS cases, indicating the presence of additional, unknown factors that could impact sTDP43 deposition or immunoreactivity.

In light of endogenous sTDP43 detected within mouse spinal cord astrocytes (Supplemental Figure 12B) and human iPSCderived astrocytes (Supplemental Figure 13), we asked if sTDP43 pathology might also be present within astrocytes. In sections from controls and sALS patients immunostained with STDP43 antibodies, neurons and glia were identified by costaining with NeuN and GFAP antibodies, respectively (Figure 8F). Cytoplasmic sTDP43 accumulations were detected in both NeuN-positive neurons and GFAP-positive astrocytes, suggesting that sTDP43 pathology is not limited to neurons. Taken together, these results demonstrate that endogenous sTDP43 accumulates within neurons and glia of individuals with ALS, supporting a potentially pathogenic contribution of sTDP43 isoforms to ALS pathogenesis.

\section{Discussion}

In this study, we show that neuronal hyperactivity leads to the selective upregulation of C-terminally truncated TDP43 isoforms (sTDP43). These isoforms are intrinsically insoluble and accumulate within cytoplasmic aggregates by virtue of an NES present within a unique 18 -amino acid C-terminus. sTDP43 also sequesters endogenous TDP43 within cytoplasmic aggregates and induces its clearance from the nucleus, thereby recapitulating signature pathologic changes found in the majority of individuals with ALS and implicating complementary gain- and loss-of-function mechanisms in disease pathogenesis. sTDP43 transcripts are enriched in spinal motor neurons, a cell type that is selectively vulnerable in ALS, and postmortem samples from individuals with ALS show conspicuous accumulations of sTDP43 within affected neurons and glia. These observations suggest a fundamental link between neuronal hyperexcitability and TDP43 pathology, 2 conserved features characteristic of both familial and sALS. Moreover, they raise the possibility that sTDP43 production and/or its accumulation are heretoforeunrecognized contributors to neurodegeneration in ALS.

A series of previous studies demonstrated that alternative $T A R D B P$ splicing gives rise to truncated TDP43 isoforms lacking the $\mathrm{C}$-terminus that are highly insoluble when overexpressed in heterologous systems $(60,64,65)$. Here, we show that neuronal activity selectively upregulates these truncated isoforms, which we collectively labeled sTDP43, despite a simultaneous increase in mRNA encoding flTDP43. This discrepancy may arise from the relative inability of sTDP43 to effectively participate in autoregulation (Supplemental Figure 7), or the presence of unique elements within the flTDP43 3'UTR leading to nuclear mRNA retention and/or destabilization $(61-63,83,84)$. As such, the activity-dependent and apparently selective upregulation of STDP43, together with the widespread 
neuronal hyperactivity observed in ALS patients, animal models, and human iPSC-derived neurons $(26,28,34,35,42,43)$, may be a crucial factor driving sTDP43 deposition in ALS tissue.

In keeping with previous studies $(64,65)$, overexpressed sTDP43 accumulates in the cytoplasm where it often forms large, insoluble inclusions. The low-complexity domain (LCD) within the TDP43 C-terminus promotes liquid-phase separation and aggregation (90-94). Even so, our observations and those of others $(64,65)$ show that sTDP43 is relatively insoluble and prone to aggregation, despite lacking the LCD. A growing body of evidence suggests that proteins with complex, folded domains such as the TDP43 RNA-recognition motifs (RRMs) are highly susceptible to aggregation (95). Rather than promoting insolubility, the presence of LCDs within these proteins protects against misfolding and aggregation by enabling reversible phase transitions during conditions of supersaturation. Thus, LCDs may permit higher local concentrations of RRM-containing proteins than would otherwise be possible without misfolding and/or aggregation (96). In this regard, the absence of the LCD may be directly responsible for the enhanced aggregation of sTDP43; indeed, several RNA-binding proteins display similar phenotypes upon removal of the LCD, including PUB1, PAB1, and SUP35 (96-99).

Using predictive software, we identified a potential NES located within the 18-amino acid sTDP43 C-terminus, and experimentally confirmed that this segment drives cytoplasmic sTDP43 localization. This NES appears to be dominant over the functional NLS present within the N-terminus of STDP43, either due to a high affinity for nuclear exporters or because of enhanced accessibility of the NES at the extreme C-terminus of the protein. The previously annotated TDP43 NES $(68,100)$ exhibits leucine-/ isoleucine-rich sequences favored by exportin-1 (XPO1), an essential mediator of protein nuclear export (101). Nevertheless, scant experimental evidence suggests that this sequence functions as a true NES. TDP43 and XPO1 do not interact with one another in vitro $(15,102)$, and unbiased proteomics studies have failed to identify TDP43 as an XPO1 cargo protein $(103,104)$. Further, TDP43 localization is unaffected by XPO1 inhibition or deletion of the putative NES (15). In contrast, the NES uncovered within the sTDP43 C-terminal tail is both necessary and sufficient for sTDP43 nuclear export, suggesting that it is a bona fide NES.

sTDP43 lacks the C-terminal glycine-rich domain required for splicing activity (105); as such, sTDP43 is incapable of CFTR minigene splicing or effectively participating in TDP43 autoregulation, which involves differential splicing of the TARDBP $3^{\prime} \mathrm{UTR}$ $(83,84)$. The C-terminal glycine-rich domain is also required for toxicity upon TDP43 overexpression in yeast (94). Nevertheless, sTDP43 overexpression was still lethal in neurons. We suspect that STDP43-related toxicity arises from a combination of factors, including (a) the NES within the new C-terminal tail region provoking cytoplasmic sTDP43 deposition; (b) its interaction with endogenous flTDP43 via its N-terminus $(52,73,106)$; and (c) the presence of intact RRMs that enable sTDP43 to bind and potentially sequester cytoplasmic mRNAs.

sTDP43 isoforms are highly conserved in humans, nonhuman primates, and lesser mammals at the transcript and protein levels. Such evolutionary conservation suggests that these isoforms fulfill unknown functions, perhaps involving a compensatory response to chronic neuronal hyperactivity or generalized stress. Intriguingly, sTDP43 transcripts are significantly enriched in murine motor neurons compared with frontal cortex homogenate, their expression increases with age, and sTDP43-1 is the dominant TARDBP species in human lumbar motor neurons, raising the possibility that spinal motor neurons accumulate potentially toxic levels of sTDP43 in response to aging and hyperexcitability. Future studies are needed to determine whether native sTDP43 performs an essential function in motor neurons or other cell types, and if sTDP43 contributes to the selective vulnerability of aged motor neurons in ALS $(107,108)$.

By creating an antibody that recognizes the unique sTDP43 C-terminus, we detected cytoplasmic sTDP43 inclusions selectively within the spinal cord and cortex of ALS patients, including individuals with sALS and C9ALS. In addition, the presence of sTDP43 deposits coincided with nuclear TDP43 exclusion, as predicted by sTDP43 nuclear export and its affinity for flTDP43. Although the aggregation-prone TDP43 C-terminus forms a core component of the cytoplasmic inclusions found in ALS patients (109-117), emerging evidence suggests that N-terminal TDP43 fragments also contribute to ALS pathogenesis. N-terminal TDP43 fragments are observed in ALS patient spinal cord $(118,119)$, and in keeping with studies of RNA-binding proteins in yeast, the TDP43 RRMs misfold and aggregate in vitro without the C-terminal LCD to maintain solubility $(65,95,97-99,120-122)$. Independent of the RRMs, the TDP43 N-terminus enhances TDP43 aggregation and toxicity $(65,79,120,121)$, potentially adding to sTDP43 insolubility and the impact of sTDP43 deposition in affected neurons.

TDP43-positive cytoplasmic inclusions in ALS are not limited to neurons but are also found in astrocytes and oligodendrocytes (123-126). Astrocytes help regulate extracellular glutamate levels, and their dysfunction in ALS may lead to impaired synaptic glutamate buffering in sALS as well as familial ALS (127-132). In addition to detecting endogenous STDP43 production in cultured human astrocytes and murine spinal cord, we noted diseasespecific astrocyte sTDP43 pathology in sALS patient tissue. Although the effect of STDP43 accumulation in these cells remains to be determined, it is possible that sTDP43-induced astrocyte toxicity triggers a feed-forward mechanism in which reduced glutamate buffering results in neuronal hyperactivity, increased sTDP43 production, and subsequent neurodegeneration.

We and others (61) have been unable to reliably detect endogenous sTDP43 isoforms by Western blotting, an effect likely related to the intrinsic insolubility of the protein $(61,65)$ (Figure 8A). In previous studies, however, tandem mass spectrometry identified $\mathrm{C}$-terminal sequences unique to sTDP43 in mouse brain tissue (61), confirming the presence of sTDP43 in the CNS. Despite the limitations of the newly developed sTDP43 antibody for Western blotting, its utility for immunofluorescence is supported by several observations. First, sTDP43 immunoreactivity varied bidirectionally in TEA- and TTX-treated iNeurons (Figure 8, B and C), analogous to the pattern detected by $\mathrm{N}$-terminal but not $\mathrm{C}$-terminal TDP43 antibodies (Figure 1, H-K). Second, endogenous TDP43 labeled with Dendra2 at the $\mathrm{N}$-terminus but not C-terminus demonstrated an identical pattern (Figure 2), and nearly all of the sTDP43 immunoreactivity in human brains overlapped with signal from an N-terminally directed TDP43 antibody (Figure 8D), as predicted for STDP43 isoforms missing the conventional C-terminus. 
Our work underlines the significance of previously identified sTDP43 isoforms and highlights a pivotal connection between neuronal hyperexcitability and TDP43 pathology, 2 conserved findings in ALS. Many questions remain, including the function of sTDP43 isoforms, the extent and pervasiveness of sTDP43 pathology in ALS, and whether cell type- or species-specific differences in STDP43 expression contribute to the selective vulnerability of human motor neurons in ALS. Complementary investigations of sTDP43 splicing and its regulation are crucial if we are to determine if targeted manipulation of STDP43 has the potential to prevent or slow motor neuron degeneration in ALS.

\section{Methods}

Materials and methods are listed in the supplemental material.

Study approval. All vertebrate animal work was approved by the Committee on the Use and Care of Animals (UCUCA) at the University of Michigan and in accordance with the United Kingdom Animals Act (1986). All experiments were performed in accordance with UCUCA guidelines. Rats (Rattus norvegicus) used for primary neuron collection were housed singly in chambers equipped with environmental enrichment. All studies were designed to minimize animal use. Rats were cared for by the Unit for Laboratory Animal Medicine at the University of Michigan; all individuals were trained and approved in the care and long-term maintenance of rodent colonies, in accordance with the NIH-supported Guide for the Care and Use of Laboratory Animals (National Academies Press, 2011). All personnel handling the rats and administering euthanasia were properly trained in accordance with the University of Michigan Policy for Education and Training of Animal Care and Use Personnel. Euthanasia was fully consistent with the recommendations of the Guidelines on Euthanasia of the American Veterinary Medical Association.

\section{Author contributions}

KW was responsible for conceptualization, methodology, investigation, formal analysis, writing, and visualization. SJB contributed to conceptualization, methodology, formal analysis, writing, visualization, supervision, project administration, and funding acquisition. EMT contributed to conceptualization and methodology, and RM was responsible for software. MW and NBG contributed to data curation and formal analysis. JS and AS contributed to supervision and project administration. JPM, ZL, CMG, and AS contributed to the investigation.

\section{Acknowledgments}

This work was supported by the NIH National Institute for Neurological Disorders and Stroke (NINDS) R01-NS097542, National Institute for Aging (NIA) P30 AG053760 (to SJB), the University of Michigan Protein Folding Disease Initiative, and Ann Arbor Active Against ALS. We thank M. Uhler for advice, protocols and reagents; M. Ward, Z. Xu, and Y. Ayala for reagents; and J. Parent, A. Malik, P. Garay, and M. McMillan for their suggestions. We also thank M. Perkins from the Michigan Brain Bank (5P30 AG053760, University of Michigan Alzheimer's Disease Core Center), the Michigan ALS Biorepository, the University of Michigan DNA Sequencing Core, and the University of Michigan Department of Pharmacology for access to their confocal microscopy core. Finally, we thank those that donated the fibroblast and tissue samples that made these studies possible.

Address correspondence to: Sami Barmada, University of Michigan, Department of Neurology, 109 Zina Pitcher Place, BSRB 4019, Ann Arbor, Michigan 48109, USA. Phone: 734.763.2624; Email: sbarmada@umich.edu.
1. Bruijn LI, Miller TM, Cleveland DW. Unraveling the mechanisms involved in motor neuron degeneration in ALS. Annu Rev Neurosci. 2004;27:723-749.

2. Neumann M. Molecular neuropathology of TDP-43 proteinopathies. Int JMol Sci. 2009;10(1):232-246.

3. Murray ME, et al. Clinical and neuropathologic heterogeneity of c9FTD/ALS associated with hexanucleotide repeat expansion in C9ORF72. Acta Neuropathol. 2011;122(6):673-690.

4. Seilhean D, et al. Accumulation of TDP-43 and alpha-actin in an amyotrophic lateral sclerosis patient with the K17I ANG mutation. Acta Neuropathol. 2009;118(4):561-573.

5. Van Mossevelde S, et al. Clinical features of TBK1 carriers compared with C9orf72, GRN and non-mutation carriers in a Belgian cohort. Brain. 2016;139(Pt 2):452-467.

6. Smith BN, et al. Novel mutations support a role for Profilin 1 in the pathogenesis of ALS. Neurobiol Aging. 2015;36(3):1602.e17-1602.e27.

7. Deng HX, et al. Mutations in UBQLN2 cause dominant X-linked juvenile and adult-onset ALS and ALS/dementia. Nature. 2011;477(7363):211-215.

8. Johnson JO, et al. Exome sequencing reveals VCP mutations as a cause of familial ALS. Neuron. 2010;68(5):857-864.

9. Kim HJ, et al. Mutations in prion-like domains in hnRNPA2B1 and hnRNPA1 cause mul- tisystem proteinopathy and ALS. Nature. 2013;495(7442):467-473.

10. Kraemer BC, et al. Loss of murine TDP-43 disrupts motor function and plays an essential role in embryogenesis. Acta Neuropathol. 2010;119(4):409-419.

11. Wu LS, Cheng WC, Shen CK. Targeted depletion of TDP-43 expression in the spinal cord motor neurons leads to the development of amyotrophic lateral sclerosis-like phenotypes in mice. $J$ Biol Chem. 2012;287(33):27335-27344.

12. Iguchi $Y$, et al. Loss of TDP-43 causes age-dependent progressive motor neuron degeneration. Brain. 2013;136(Pt 5):1371-1382.

13. Sephton CF, et al. TDP-43 is a developmentally regulated protein essential for early embryonic development. J Biol Chem. 2010;285(9):6826-6834.

14. Barmada SJ, Skibinski G, Korb E, Rao EJ, Wu JY, Finkbeiner S. Cytoplasmic mislocalization of TDP-43 is toxic to neurons and enhanced by a mutation associated with familial amyotrophic lateral sclerosis. J Neurosci. 2010;30(2):639-649.

15. Archbold HC, et al. TDP43 nuclear export and neurodegeneration in models of amyotrophic lateral sclerosis and frontotemporal dementia. Sci Rep. 2018;8(1):4606.

16. Swarup V, et al. Pathological hallmarks of amyotrophic lateral sclerosis/frontotemporal lobar degeneration in transgenic mice pro- duced with TDP-43 genomic fragments. Brain. 2011;134(Pt 9):2610-2626.

17. Wils H, et al. TDP-43 transgenic mice develop spastic paralysis and neuronal inclusions characteristic of ALS and frontotemporal lobar degeneration. Proc Natl Acad Sci USA. 2010;107(8):3858-3863.

18. Dayton RD, et al. Selective forelimb impairment in rats expressing a pathological TDP- $4325 \mathrm{kDa}$ C-terminal fragment to mimic amyotrophic lateral sclerosis. Mol Ther. 2013;21(7):1324-1334.

19. Tatom JB, et al. Mimicking aspects of frontotemporal lobar degeneration and Lou Gehrig's disease in rats via TDP-43 overexpression. Mol Ther. 2009;17(4):607-613.

20 . Voigt A, et al. TDP-43-mediated neuron loss in vivo requires RNA-binding activity. PLoS One. 2010;5(8):e12247.

21. Li Y, et al. A Drosophila model for TDP43 proteinopathy. Proc Natl Acad Sci USA. 2010;107(7):3169-3174.

22. Kabashi E, et al. Gain and loss of function of ALS-related mutations of TARDBP (TDP-43) cause motor deficits in vivo. Hum Mol Genet. 2010;19(4):671-683.

23. Schmid B, et al. Loss of ALS-associated TDP43 in zebrafish causes muscle degeneration, vascular dysfunction, and reduced motor neuron axon outgrowth. Proc Natl Acad Sci USA. 2013;110(13):4986-4991. 
24. Uchida A, et al. Non-human primate model of amyotrophic lateral sclerosis with cytoplasmic mislocalization of TDP-43. Brain. 2012;135(Pt 3):833-846.

25. Jackson $\mathrm{KL}$, et al. Initial gene vector dosing for studying symptomatology of amyotrophic lateral sclerosis in non-human primates. JMed Primatol. 2015;44(2):66-75.

26. Vucic S, Nicholson GA, Kiernan MC. Cortical hyperexcitability may precede the onset of familial amyotrophic lateral sclerosis. Brain. 2008;131(Pt 6):1540-1550.

27. Kanai K, et al. Motor axonal excitability properties are strong predictors for survival in amyotrophic lateral sclerosis. J Neurol Neurosurg Psychiatry. 2012;83(7):734-738.

28. Kanai K, et al. Altered axonal excitability properties in amyotrophic lateral sclerosis: impaired potassium channel function related to disease stage. Brain. 2006;129(Pt 4):953-962.

29. Enterzari-Taher M, Eisen A, Stewart H, Nakajima M. Abnormalities of cortical inhibitory neurons in amyotrophic lateral sclerosis. Muscle Nerve. 1997;20(1):65-71.

30. Zanette G, Tamburin S, Manganotti P, Refatti N, Forgione A, Rizzuto N. Different mechanisms contribute to motor cortex hyperexcitability in amyotrophic lateral sclerosis. Clin Neurophysiol. 2002;113(11):1688-1697.

31. Geevasinga N, et al. Cortical function in asymptomatic carriers and patients with C9orf72 amyotrophic lateral sclerosis. JAMA Neurol. 2015;72(11):1268-1274.

32. Vucic S, Cheah BC, Kiernan MC. Defining the mechanisms that underlie cortical hyperexcitability in amyotrophic lateral sclerosis. Exp Neurol.2009;220(1):177-182.

33. Karandreas N, Papadopoulou M, Kokotis P, Papapostolou A, Tsivgoulis G, Zambelis T. Impaired interhemispheric inhibition in amyotrophic lateral sclerosis. Amyotroph Lateral Scler. 2007;8(2):112-118.

34. Tamura $\mathrm{N}$, et al. Increased nodal persistent $\mathrm{Na}^{+}$ currents in human neuropathy and motor neuron disease estimated by latent addition. Clin Neurophysiol. 2006;117(11):2451-2458.

35 . Shibuya K, et al. Markedly reduced axonal potassium channel expression in human sporadic amyotrophic lateral sclerosis: an immunohistochemical study. Exp Neurol. 2011;232(2):149-153.

36. Geevasinga $\mathrm{N}$, et al. Riluzole exerts transient modulating effects on cortical and axonal hyperexcitability in ALS. Amyotroph Lateral Scler Frontotemporal Degener. 2016;17(7-8):580-588.

37. Kuo JJ, et al. Hyperexcitability of cultured spinal motoneurons from presymptomatic ALS mice. J Neurophysiol. 2004;91(1):571-575.

38. Pieri M, et al. Altered excitability of motor neurons in a transgenic mouse model of familial amyotrophic lateral sclerosis. Neurosci Lett. 2003;351(3):153-156.

39. van Zundert B, et al. Neonatal neuronal circuitry shows hyperexcitable disturbance in a mouse model of the adult-onset neurodegenerative disease amyotrophic lateral sclerosis. J Neurosci. 2008;28(43):10864-10874.

40. Saba L, et al. Altered functionality, morphology, and vesicular glutamate transporter expression of cortical motor neurons from a presymptomatic mouse model of amyotrophic lateral sclerosis. Cereb Cortex. 2016;26(4):1512-1528.

41. Pieri M, Carunchio I, Curcio L, Mercuri NB, Zona C. Increased persistent sodium current determines cortical hyperexcitability in a genetic model of amyotrophic lateral sclerosis. Exp Neurol. 2009;215(2):368-379.

42. Wainger BJ, et al. Intrinsic membrane hyperexcitability of amyotrophic lateral sclerosis patient-derived motor neurons. Cell Rep. 2014;7(1):1-11.

43. Devlin AC, et al. Human iPSC-derived motoneurons harbouring TARDBP or C9ORF72 ALS mutations are dysfunctional despite maintaining viability. Nat Commun. 2015;6:5999.

44. Sareen D, et al. Targeting RNA foci in iPSC-derived motor neurons from ALS patients with a C9orf72 repeat expansion. Sci Transl Med. 2013;5(208):208ra149.

45. Zhang Y, et al. Rapid single-step induction of functional neurons from human pluripotent stem cells. Neuron. 2013;78(5):785-798.

46. Lam RS, Töpfer FM, Wood PG, Busskamp V, Bamberg E. Functional maturation of human stem cell-derived neurons in long-term cultures. PLoS One. 2017;12(1):e0169506.

47. Busskamp V, et al. Rapid neurogenesis through transcriptional activation in human stem cells. Mol Syst Biol. 2014;10:760.

48. Gupta S, et al. Fibroblast growth factor 2 regulates activity and gene expression of human post-mitotic excitatory neurons. J Neurochem. 2018;145(3):188-203.

49. Wang IF, Wu LS, Chang HY, Shen CK. TDP-43, the signature protein of FTLD-U, is a neuronal activity-responsive factor. J Neurochem. 2008;105(3):797-806

50. Schwenk BM, et al. TDP-43 loss of function inhibits endosomal trafficking and alters trophic signaling in neurons. EMBO J. 2016;35(21):2350-2370.

51. Gopal PP, Nirschl JJ, Klinman E, Holzbaur EL. Amyotrophic lateral sclerosis-linked mutations increase the viscosity of liquid-like TDP-43 RNP granules in neurons. Proc Natl Acad Sci USA. 2017;114(12):E2466-E2475.

52. Zhang YJ, et al. The dual functions of the extreme $\mathrm{N}$-terminus of TDP-43 in regulating its biological activity and inclusion formation. Hum Mol Genet. 2013;22(15):3112-3122.

53. Suzuki H, Shibagaki Y, Hattori S, Matsuoka M. Nuclear TDP- 43 causes neuronal toxicity by escaping from the inhibitory regulation by hnRNPs. Hum Mol Genet. 2015;24(6):1513-1527.

54. Baker M. Reproducibility crisis: Blame it on the antibodies. Nature. 2015;521(7552):274-276.

55. Begley CG, Ellis LM. Drug development: Raise standards for preclinical cancer research. Nature 2012;483(7391):531-533.

56. Ran FA, et al. Double nicking by RNA-guided CRISPR Cas 9 for enhanced genome editing specificity. Cell. 2013;154(6):1380-1389.

57. Eom T, et al. NOVA-dependent regulation of cryptic NMD exons controls synaptic protein levels after seizure. Elife. 2013;2:e00178.

58. Kolisnyk B, et al. Cholinergic regulation of hnRN$\mathrm{PA} 2 / \mathrm{B} 1$ translation by M1 muscarinic receptors. J Neurosci. 2016;36(23):6287-6296.
59. Flores BN, Li X, Malik AM, Martinez J, Beg AA, Barmada SJ. An intramolecular salt bridge linking TDP43 RNA binding, protein stability, and TDP43-dependent neurodegeneration. Cell Rep. 2019;27(4):1133-1150.e8.

60. Wang HY, Wang IF, Bose J, Shen CK. Structural diversity and functional implications of the eukaryotic TDP gene family. Genomics. 2004;83(1):130-139.

61. D'Alton S, Altshuler M, Lewis J. Studies of alternative isoforms provide insight into TDP43 autoregulation and pathogenesis. RNA. 2015;21(8):1419-1432.

62. Avendaño-Vázquez SE, Dhir A, Bembich S, Buratti E, Proudfoot N, Baralle FE. Autoregulation of TDP-43 mRNA levels involves interplay between transcription, splicing, and alternative polyA site selection. Genes Dev. 2012;26(15):1679-1684.

63. Bembich S, et al. Predominance of spliceosomal complex formation over polyadenylation site selection in TDP-43 autoregulation. Nucleic Acids Res. 2014;42(5):3362-3371.

64. Seyfried NT, et al. Multiplex SILAC analysis of a cellular TDP-43 proteinopathy model reveals protein inclusions associated with SUMOylation and diverse polyubiquitin chains. Mol Cell Proteomics. 2010;9(4):705-718.

65. Dammer EB, et al. Coaggregation of RNA-binding proteins in a model of TDP-43 proteinopathy with selective RGG motif methylation and a role for RRM1 ubiquitination. PLoS One. 2012;7(6):e38658.

66. la Cour T, Kiemer L, Mølgaard A, Gupta R, Skriver K, Brunak S. Analysis and prediction of leucine-rich nuclear export signals. Protein Eng Des Sel. 2004;17(6):527-536.

67. Niopek D, Wehler P, Roensch J, Eils R, Di Ventura B. Optogenetic control of nuclear protein export. Nat Commun. 2016;7:10624.

68. Ayala YM, et al. Structural determinants of the cellular localization and shuttling of TDP-43. J Cell Sci. 2008;121(Pt 22):3778-3785.

69. Barmada SJ, et al. Amelioration of toxicity in neuronal models of amyotrophic lateral sclerosis by hUPF1. Proc Natl Acad Sci USA. 2015;112(25):7821-7826.

70. Malik AM, Miguez RA, Li X, Ho YS, Feldman EL, Barmada SJ. Matrin 3-dependent neurotoxicity is modified by nucleic acid binding and nucleocytoplasmic localization. Elife. 2018;7:e35977.

71. Weskamp K, Safren N, Miguez R, Barmada S. Monitoring neuronal survival via longitudinal fluorescence microscopy. J Vis Exp. 2019;(143):59036.

72. Christensen E. Multivariate survival analysis using Cox's regression model. Hepatology. 1987;7(6):1346-1358.

73. Afroz T, et al. Functional and dynamic polymerization of the ALS-linked protein TDP-43 antagonizes its pathologic aggregation. Nat Commun . 2017;8(1):45.

74. Kuo PH, Doudeva LG, Wang YT, Shen CK, Yuan HS. Structural insights into TDP-43 in nucleic-acid binding and domain interactions. Nucleic Acids Res. 2009;37(6):1799-1808.

75. Johnson BS, Snead D, Lee JJ, McCaffery JM, Shorter J, Gitler AD. TDP- 43 is intrinsically aggregation-prone, and amyotrophic lateral 
sclerosis-linked mutations accelerate aggregation and increase toxicity. J Biol Chem. 2009;284(30):20329-20339.

76. Zhang YJ, et al. Aberrant cleavage of TDP-43 enhances aggregation and cellular toxicity. Proc Natl Acad Sci USA . 2009;106(18):7607-7612.

77. Bozzo F, et al. Structural insights into the multi-determinant aggregation of TDP-43 in motor neuron-like cells. Neurobiol Dis. 2016;94:63-72.

78. Budini M, Romano V, Quadri Z, Buratti E, Baralle FE. TDP-43 loss of cellular function through aggregation requires additional structural determinants beyond its C-terminal Q/N prion-like domain. Hum Mol Genet. 2015;24(1):9-20.

79. Chang CK, et al. The N-terminus of TDP-43 promotes its oligomerization and enhances DNA binding affinity. Biochem Biophys Res Commun . 2012;425(2):219-224.

80. Arrasate M, Mitra S, Schweitzer ES, Segal MR, Finkbeiner S. Inclusion body formation reduces levels of mutant huntingtin and the risk of neuronal death. Nature. 2004;431(7010):805-810.

81. Ayala YM, Pagani F, Baralle FE. TDP43 depletion rescues aberrant CFTR exon 9 skipping. FEBS Lett. 2006;580(5):1339-1344.

82. Buratti E, Dörk T, Zuccato E, Pagani F, Romano M, Baralle FE. Nuclear factor TDP-43 and SR proteins promote in vitro and in vivo CFTR exon 9 skipping. EMBO J. 2001;20(7):1774-1784.

83. Polymenidou $\mathrm{M}$, et al. Long pre-mRNA depletion and RNA missplicing contribute to neuronal vulnerability from loss of TDP-43. Nat Neurosci. 2011;14(4):459-468.

84. Ayala YM, et al. TDP- 43 regulates its mRNA levels through a negative feedback loop. EMBO J. 2011;30(2):277-288.

85. White MA, et al. TDP-43 gains function due to perturbed autoregulation in a Tardbp knockin mouse model of ALS-FTD. Nat Neurosci. 2018;21(4):552-563.

86. Krach F, et al. Transcriptome-pathology correlation identifies interplay between TDP- 43 and the expression of its kinase CK1E in sporadic ALS. Acta Neuropathol. 2018;136(3):405-423.

87. D'Erchia AM, et al. Massive transcriptome sequencing of human spinal cord tissues provides new insights into motor neuron degeneration in ALS. Sci Rep. 2017;7(1):10046.

88. Prudencio M, et al. Distinct brain transcriptome profiles in C9orf72-associated and sporadic ALS. Nat Neurosci. 2015;18(8):1175-1182.

89. Yang C, et al. Partial loss of TDP-43 function causes phenotypes of amyotrophic lateral sclerosis. Proc Natl Acad Sci USA. 2014;111(12):E1121-E1129.

90. Li HR, Chiang WC, Chou PC, Wang WJ, Huang JR. TAR DNA-binding protein 43 (TDP-43) liquid-liquid phase separation is mediated by just a few aromatic residues. J Biol Chem. 2018;293(16):6090-6098.

91. McGurk L, et al. Poly(ADP-ribose) prevents pathological phase separation of TDP- 43 by promoting liquid demixing and stress granule localization. Mol Cell. 2018;71(5):703-717.e9.

92. Conicella AE, Zerze GH, Mittal J, Fawzi NL. ALS mutations disrupt phase separation mediated by $\alpha$-helical structure in the TDP- 43 low-complexity C-terminal domain. Structure.
2016;24(9):1537-1549.

93. Mann JR, et al. RNA binding antagonizes neurotoxic phase transitions of TDP-43. Neuron. 2019;102(2):321-338.e8.

94. Johnson BS, McCaffery JM, Lindquist S, Gitler AD. A yeast TDP-43 proteinopathy model: Exploring the molecular determinants of TDP-43 aggregation and cellular toxicity. Proc Natl Acad Sci USA. 2008;105(17):6439-6444.

95. Agrawal S, Kuo PH, Chu LY, Golzarroshan B, Jain M, Yuan HS. RNA recognition motifs of disease-linked RNA-binding proteins contribute to amyloid formation. Sci Rep. 2019;9(1):6171.

96. Franzmann TM, Alberti S. Prion-like low-complexity sequences: Key regulators of protein solubility and phase behavior. J Biol Chem. 2019;294(18):7128-7136.

97. Riback JA, et al. Stress-triggered phase separation is an adaptive, evolutionarily tuned response. Cell. 2017;168(6):1028-1040.e19.

98. Kroschwald S, et al. Different material states of Pub1 condensates define distinct modes of stress adaptation and recovery. Cell Rep. 2018;23(11):3327-3339.

99. Franzmann TM, et al. Phase separation of a yeast prion protein promotes cellular fitness. Science. 2018;359(6371):eaao5654.

100.Winton MJ, Igaz LM, Wong MM, Kwong LK, Trojanowski JQ, Lee VM. Disturbance of nuclear and cytoplasmic TAR DNA-binding protein (TDP43) induces disease-like redistribution, sequestration, and aggregate formation. J Biol Chem. 2008;283(19):13302-13309.

101. Fornerod M, Ohno M, Yoshida M, Mattaj IW. CRM1 is an export receptor for leucine-rich nuclear export signals. Cell.1997;90(6):1051-1060.

102. Pinarbasi ES, Cağatay T, Fung HYJ, Li YC, Chook YM, Thomas PJ. Active nuclear import and passive nuclear export are the primary determinants of TDP-43 localization. Sci Rep. 2018;8(1):7083.

103. Kırlı K, et al. A deep proteomics perspective on CRM1-mediated nuclear export and nucleocytoplasmic partitioning. Elife. 2015;4:e11466.

104. Thakar K, Karaca S, Port SA, Urlaub H, Kehlenbach RH. Identification of CRM1dependent nuclear export cargos using quantitative mass spectrometry. Mol Cell Proteomics. 2013;12(3):664-678.

105. Ayala YM, et al. Human, Drosophila, and C.elegans TDP43: nucleic acid binding properties and splicing regulatory function. J Mol Biol. 2005;348(3):575-588.

106.Wang A, et al. A single N-terminal phosphomimic disrupts TDP- 43 polymerization, phase separation, and RNA splicing. EMBOJ. 2018;37(5):e97452.

107. Leigh PN, et al. Ubiquitin-immunoreactive intraneuronal inclusions in amyotrophic lateral sclerosis. Morphology, distribution, and specificity. Brain. 1991;114(Pt 2):775-788.

108. Ravits J, Paul P, Jorg C. Focality of upper and lower motor neuron degeneration at the clinical onset of ALS. Neurology. 2007;68(19):1571-1575.

109. Udan M, Baloh RH. Implications of the prionrelated Q/N domains in TDP-43 and FUS. Prion. 2011;5(1):1-5.

110. Furukawa Y, Kaneko K, Watanabe S, Yamanaka $\mathrm{K}$, Nukina N. A seeding reaction recapitulates intracellular formation of Sarkosyl-insoluble transactivation response element (TAR) DNA-binding protein-43 inclusions. J Biol Chem. 2011;286(21):18664-18672.

111. Nonaka T, Kametani F, Arai T, Akiyama H, Hasegawa M. Truncation and pathogenic mutations facilitate the formation of intracellular aggregates of TDP-43. Hum Mol Genet. 2009;18(18):3353-3364.

112. Tsuji H, et al. Molecular analysis and biochemical classification of TDP-43 proteinopathy. Brain. 2012;135(Pt 11):3380-3391.

113. Neumann M, et al. Ubiquitinated TDP-43 in frontotemporal lobar degeneration and amyotrophic lateral sclerosis. Science. 2006;314(5796):130-133.

114. Igaz LM, et al. Expression of TDP-43 C-terminal fragments in vitro recapitulates pathological features of TDP-43 proteinopathies. J Biol Chem. 2009;284(13):8516-8524

115. Wang IF, Chang HY, Hou SC, Liou GG, Way TD, James Shen CK. The self-interaction of native TDP-43 C terminus inhibits its degradation and contributes to early proteinopathies. Nat Commun. 2012;3:766.

116. Wang YT, et al. The truncated C-terminal RNA recognition motif of TDP- 43 protein plays a key role in forming proteinaceous aggregates. J Biol Chem. 2013;288(13):9049-9057.

117. Berning BA, Walker AK. The pathobiology of TDP-43 C-terminal fragments in ALS and FTLD. Front Neurosci. 2019;13:335.

118. Yamashita T, et al. A role for calpain-dependent cleavage of TDP-43 in amyotrophic lateral sclerosis pathology. Nat Commun. 2012;3:1307.

119. Igaz LM, et al. Enrichment of C-terminal fragments in TAR DNA-binding protein- 43 cytoplasmic inclusions in brain but not in spinal cord of frontotemporal lobar degeneration and amyotrophic lateral sclerosis. Am J Pathol. 2008;173(1):182-194.

120.Zacco E, Martin SR, Thorogate R, Pastore A. The RNA-recognition motifs of TAR DNA-binding protein 43 may play a role in the aberrant self-assembly of the protein. Front Mol Neurosci. 2018;11:372.

121. Sasaguri $\mathrm{H}$, et al. The extreme $\mathrm{N}$-terminus of TDP-43 mediates the cytoplasmic aggregation of TDP-43 and associated toxicity in vivo. Brain Res. 2016;1647:57-64.

122. Shodai A, et al. Aberrant assembly of RNA recognition motif 1 links to pathogenic conversion of TAR DNA-binding protein of $43 \mathrm{kDa}$ (TDP-43). J Biol Chem. 2013;288(21):14886-14905.

123. Neumann M, et al. TDP-43-positive white matter pathology in frontotemporal lobar degeneration with ubiquitin-positive inclusions. J Neuropathol Exp Neurol. 2007;66(3):177-183.

124. Nishihira Y, et al. Sporadic amyotrophic lateral sclerosis: two pathological patterns shown by analysis of distribution of TDP-43-immunoreactive neuronal and glial cytoplasmic inclusions. Acta Neuropathol. 2008;116(2):169-182.

125. Zhang H, et al. TDP-43-immunoreactive neuronal and glial inclusions in the neostriatum in amyotrophic lateral sclerosis with and without dementia. Acta Neuropathol. 2008;115(1):115-122.

126. Serio A, et al. Astrocyte pathology and the absence of non-cell autonomy in an induced pluripotent 
stem cell model of TDP-43 proteinopathy. Proc Natl Acad Sci USA. 2013;110(12):4697-4702.

127. Bruijn LI, et al. ALS-linked SOD1 mutant G85R mediates damage to astrocytes and promotes rapidly progressive disease with SOD1-containing inclusions. Neuron. 1997;18(2):327-338.

128.Guo H, et al. Increased expression of the glial glutamate transporter EAAT2 modulates excitotoxicity and delays the onset but not the outcome of ALS in mice. Hum Mol Genet. 2003;12(19):2519-2532.

129. Howland DS, et al. Focal loss of the glutamate transporter EAAT2 in a transgenic rat model of SOD1 mutant-mediated amyotrophic lateral sclerosis (ALS). Proc Natl Acad Sci USA. 2002;99(3):1604-1609.

130. Pardo AC, et al. Loss of the astrocyte glutamate transporter GLT1 modifies disease in SOD1
(G93A) mice. Exp Neurol. 2006;201(1):120-130. 131. Rothstein JD, Martin LJ, Kuncl RW. Decreased glutamate transport by the brain and spinal cord in amyotrophic lateral sclerosis. $N$ Engl J Med. 1992;326(22):1464-1468.

132. Rothstein JD, Van Kammen M, Levey AI, Martin LJ, Kuncl RW. Selective loss of glial glutamate transporter GLT-1 in amyotrophic lateral sclerosis. Ann Neurol. 1995;38(1):73-84. 